\title{
How to Improve Bayesian Reasoning Without Instruction: Frequency Formats
}

\author{
Gerd Gigerenzer \\ University of Chicago
}

\author{
Ulrich Hoffrage \\ Max Planck Institute for Psychological Research
}

\begin{abstract}
Is the mind, by design, predisposed against performing Bayesian inference? Previous research on base rate neglect suggests that the mind lacks the appropriate cognitive algorithms. However, any claim against the existence of an algorithm, Bayesian or otherwise, is impossible to evaluate unless one specifies the information format in which it is designed to operate. The authors show that Bayesian algorithms are computationally simpler in frequency formats than in the probability formats used in previous research. Frequency formats correspond to the sequential way information is acquired in natural sampling, from animal foraging to neural networks. By analyzing several thousand solutions to Bayesian problems, the authors found that when information was presented in frequency formats, statistically naive participants derived up to $50 \%$ of all inferences by Bayesian algorithms. Non-Bayesian algorithms included simple versions of Fisherian and Neyman-Pearsonian inference.
\end{abstract}

Is the mind, by design, predisposed against performing Bayesian inference? The classical probabilists of the Enlightenment, including Condorcet, Poisson, and Laplace, equated probability theory with the common sense of educated people, who were known then as "hommes éclairés." Laplace (1814/ $1951)$ declared that "the theory of probability is at bottom nothing more than good sense reduced to a calculus which evaluates that which good minds know by a sort of instinct, without being able to explain how with precision" (p. 196). The available mathematical tools, in particular the theorems of Bayes and Bernoulli, were seen as descriptions of actual human judgment (Daston, 1981, 1988). However, the years of political upheaval during the French Revolution prompted Laplace, unlike earlier writers such as Condorcet, to issue repeated disclaimers that probability theory, because of the interference of passion and desire, could not account for all relevant factors in human judgment. The Enlightenment view-that the laws of probability are the laws of the mind-moderated as it was through the French Revolution, had a profound influence on 19th- and 20th-century science. This view became the starting point for seminal contributions to mathematics, as when George Boole

Gerd Gigerenzer, Department of Psychology, University of Chicago; Ulrich Hoffrage, Max Planck Institute for Psychological Research, Munich, Germany.

This research was supported by a University of Chicago School Mathematics Project grant from the University of Chicago, by the Deutsche Forschungsgemeinschaft (HE 1491/2-2), and by the Fonds zur Förderung der wissenschaftlichen Forschung (P8842-MED), Austria. We are indebted to Gernot Kleiter, whose ideas have inspired our thinking, and to Winfried Kain, Horst Kilcher, and the late Jörg Quarder, who helped us to collect and analyze the data. We thank Valerie Chase, Lorraine Daston, Berna Eden, Ward Edwards, Klaus Fiedler, Dan Goldstein, Wolfgang Hell, Ralph Hertwig, Albert Madansky, Barbara Mellers, Peter Sedlmeier, and Bill Wimsatt for their comments on earlier versions of this article.

Correspondence concerning this article should be addressed to Gerd Gigerenzer, who is now at the Max Planck Institute for Psychological Research, Leopoldstrasse 24, 80802 Munich, Germany.
(1854/1958) derived the laws of algebra, logic, and probability from what he believed to be the laws of thought. It also became the basis of vital contributions to psychology, as when Piaget and Inhelder (1951/1975) added an ontogenetic dimension to their Enlightenment view of probabilistic reasoning. And it became the foundation of contemporary notions of rationality in philosophy and economics (e.g., Allais, 1953; L. J. Cohen, 1986).

Ward Edwards and his colleagues (Edwards, 1968; Phillips \& Edwards, 1966; and earlier, Rouanet, 1961) were the first to test experimentally whether human inference follows Bayes' theorem. Edwards concluded that inferences, although "conservative," were usually proportional to those calculated from Bayes' theorem. Kahneman and Tversky (1972, p. 450), however, arrived at the opposite conclusion: "In his evaluation of evidence, man is apparently not a conservative Bayesian: he is not Bayesian at all." In the 1970s and 1980s, proponents of their "heuristics-and-biases" program concluded that people systematically neglect base rates in Bayesian inference problems. "The genuineness, the robustness, and the generality of the base-rate fallacy are matters of established fact" (Bar-Hillel, 1980, p. 215). Bayes' theorem, like Bernoulli's theorem, was no longer thought to describe the workings of the mind. But passion and desire were no longer blamed as the causes of the disturbances. The new claim was stronger. The discrepancies were taken as tentative evidence that "people do not appear to follow the calculus of chance or the statistical theory of prediction" (Kahneman \& Tversky, 1973, p. 237). It was proposed that as a result of "limited information-processing abilities" (Lichtenstein, Fischhoff \& Phillips, 1982, p. 333), people are doomed to compute the probability of an event by crude, nonstatistical rules such as the "representativeness heuristic." Blunter still, the paleontologist Stephen J. Gould summarized what has become the common wisdom in and beyond psychology: "Tversky and Kahneman argue, correctly I think, that our minds are not built (for whatever reason) to work by the rules of probability" (Gould, 1992, p. 469).

Here is the problem. There are contradictory claims as to 
whether people naturally reason according to Bayesian inference. The two extremes are represented by the Enlightenment probabilists and by proponents of the heuristics-and-biases program. Their conflict cannot be resolved by finding further examples of good or bad reasoning; text problems generating one or the other can always be designed. Our particular difficulty is that after more than two decades of research, we still know little about the cognitive processes underlying human inference, Bayesian or otherwise. This is not to say that there have been no attempts to specify these processes. For instance, it is understandable that when the "representativeness heuristic" was first proposed in the early 1970 s to explain base rate neglect, it was only loosely defined. Yet at present, representativeness remains a vague and ill-defined notion (Gigerenzer \& Murray, 1987; Shanteau, 1989; Wallsten, 1983). For some time it was hoped that factors such as "concreteness," "vividness," "causality," "salience," "specificity," "extremeness," and "relevance" of base rate information would be adequate to explain why base rate neglect seemed to come and go (e.g., Ajzen, 1977; BarHillel, 1980; Borgida \& Brekke, 1981). However, these factors have led neither to an integrative theory nor even to specific models of underlying processes (Hammond, 1990; Koehler, in press; Lopes, 1991; Scholz, 1987).

Some have suggested that there is perhaps something to be said for both sides, that the truth lies somewhere in the middle: Maybe the mind does a little of both Bayesian computation and quick-and-dirty inference. This compromise avoids the polarization of views but makes no progress on the theoretical front.

In this article, we argue that both views are based on an incomplete analysis: They focus on cognitive processes, Bayesian or otherwise, without making the connection between what we will call a cognitive algorithm and an information format. We (a) provide a theoretical framework that specifies why frequency formats should improve Bayesian reasoning and (b) present two studies that test whether they do. Our goal is to lead research on Bayesian inference out of the present conceptual cul-de-sac and to shift the focus from human errors to human engineering (see Edwards \& von Winterfeldt, 1986): how to help people reason the Bayesian way without even teaching them.

\section{Algorithms Are Designed for Information Formats}

Our argument centers on the intimate relationship between a cognitive algorithm and an information format. This point was made in a more general form by the physicist Richard Feynman. In his classic The Character of Physical Law, Feynman (1967) placed a great emphasis on the importance of deriving different formulations for the same physical law, even if they are mathematically equivalent (e.g., Newton's law, the local field method, and the minimum principle). Different representations of a physical law, Feynman reminded us, can evoke varied mental pictures and thus assist in making new discoveries: "Psychologically they are different because they are completely unequivalent when you are trying to guess new laws" (p. 53). We agree with Feynman. The assertion that mathematically equivalent representations can make a difference to human understanding is the key to our analysis of intuitive Bayesian inference.

We use the general term information representation and the specific terms information format and information menu to refer to external representations, recorded on paper or on some other physical medium. Examples are the various formulations of physical laws included in Feynman's book and the Feynman diagrams. External representations need to be distinguished from the internal representations stored in human minds, whether the latter are propositional (e.g., Pylyshyn, 1973) or pictorial (e.g., Kosslyn \& Pomerantz, 1977). In this article, we do not make specific claims about internal representations, although our results may be of relevance to this issue.

Consider numerical information as one example of external representations. Numbers can be represented in Roman, Arabic, and binary systems, among others. These representations can be mapped one to one onto each other and are in this sense mathematically equivalent. But the form of representation can make a difference for an algorithm that does, say, multiplication. The algorithms of our pocket calculators are tuned to Arabic numbers as input data and would fail badly if one entered binary numbers. Similarly, the arithmetic algorithms acquired by humans are designed for particular representations (Stigler, 1984). Contemplate for a moment long division in Roman numerals.

Our general argument is that mathematically equivalent representations of information entail algorithms that are not necessarily computationally equivalent (although these algorithms are mathematically equivalent in the sense that they produce the same outcomes; see Larkin \& Simon, 1987; Marr, 1982). This point has an important corollary for research on inductive reasoning. Suppose we are interested in figuring out what algorithm a system uses. We will not detect the algorithm if the representation of information we provide the system does not match the representation with which the algorithm works. For instance, assume that in an effort to find out whether a system has an algorithm for multiplication, we feed that system Roman numerals. The observation that the system produces mostly garbage does not entail the conclusion that it lacks an algorithm for multiplication. We now apply this argument to Bayesian inference.

\section{Standard Probability Format}

In this article, we focus on an elementary form of Bayesian inference. The task is to infer a single-point estimate-a probability ("posterior probability") or a frequency-for one of two mutually exclusive and exhaustive hypotheses, based on one observation (rather than two or more). This elementary task has been the subject of almost all experimental studies on Bayesian inference in the last 25 years. The following "mammography problem" (adapted from Eddy, 1982) is one example:

Mammography problem (standard probability format)
The probability of breast cancer is $1 \%$ for a woman at age forty who
participates in routine screening. If a woman has breast cancer, the
probability is $80 \%$ that she will get a positive mammography. If a
woman does not have breast cancer, the probability is $9.6 \%$ that she
will also get a positive mammography. A woman in this age group
had a positive mammography in a routine screening. What is the
probability that she actually has breast cancer?

There are two mutually exclusive and exhaustive hypotheses (breast cancer and no breast cancer), there is one observation 
(the positive test), and the task is to arrive at a single-point probability estimate.

The information is represented here in terms of single-event probabilities: All information (base rate, hit rate, and false alarm rate) is in the form of probabilities attached to a single person, and the task is to estimate a single-event probability. The probabilities are expressed as percentages; alternatively, they can be presented as numbers between zero and one. We refer to this representation (base rate, hit rate, and false alarm rate expressed as single-event probabilities) as the standard probability format.

What is the algorithm needed to calculate the Bayesian posterior probability $p$ (cancer/positive) from the standard probability format? Here and in what follows, we use the symbols $H$ and $-H$ for the two hypotheses or possible outcomes (breast cancer and no breast cancer) and $D$ for the data obtained (positive mammography). A Bayesian algorithm for computing the posterior probability $p(H \mid D)$ with the values given in the standard probability format amounts to solving the following equation:

$$
\begin{aligned}
p(H \mid D) & =\frac{p(H) p(D \mid H)}{p(H) p(D \mid H)+p(-H) p(D \mid-H)} \\
& =\frac{(.01)(.80)}{(.01)(.80)+(.99)(.096)} .
\end{aligned}
$$

The result is $\mathbf{0 7 8}$. We know from several studies that physicians, college students (Eddy, 1982), and staff at Harvard Medical School (Casscells, Schoenberger \& Grayboys, 1978) all have equally great difficulties with this and similar medical disease problems. For instance, Eddy (1982) reported that 95 out of 100 physicians estimated the posterior probability $p$ (cancer $\mid$ positive) to be between $70 \%$ and $80 \%$, rather than $7.8 \%$.

The experimenters who have amassed the apparently damning body of evidence that humans fail to meet the norms of Bayesian inference have usually given their research participants information in the standard probability format (or its variant, in which one or more of the three percentages are relative frequencies; see below). Studies on the cab problem (BarHillel, 1980; Tversky \& Kahneman, 1982), the light-bulb problem (Lyon \& Slovic, 1976), and various disease problems (Casscells et al., 1978; Eddy, 1982; Hammerton, 1973) are examples. Results from these and other studies have generally been taken as evidence that the human mind does not reason with Bayesian algorithms. Yet this conclusion is not warranted, as explained before. One would be unable to detect a Bayesian algorithm within a system by feeding it information in a representation that does not match the representation with which the algorithm works.

In the last few decades, the standard probability format has become a common way to communicate information ranging from medical and statistical textbooks to psychological experiments. But we should keep in mind that it is only one of many mathematically equivalent ways of representing information; it is, moreover, a recently invented notation. Neither the standard probability format nor Equation 1 was used in Bayes' (1763) original essay. Indeed, the notion of "probability" did not gain prominence in probability theory until one century after the mathematical theory of probability was invented (Gigerenzer,
Swijtink, Porter, Daston, Beatty, \& Krüger, 1989). Percentages became common notations only during the 19 th century (mainly for interest and taxes), after the metric system was introduced during the French Revolution. Thus, probabilities and percentages took millennia of literacy and numeracy to evolve; organisms did not acquire information in terms of probabilities and percentages until very recently. How did organisms acquire information before that time? We now investigate the links between information representation and information acquisition.

\section{Natural Sampling of Frequencies}

Evolutionary theory asserts that the design of the mind and its environment evolve in tandem. Assume-pace Gould-that humans have evolved cognitive algorithms that can perform statistical inferences. These algorithms, however, would not be tuned to probabilities or percentages as input format, as explained before. For what information format were these algorithms designed? We assume that as humans evolved, the "natural" format was frequencies as actually experienced in a series of events, rather than probabilities or percentages (Cosmides \& Tooby, in press; Gigerenzer, 1991b, 1993a). From animals to neural networks, systems seem to learn about contingencies through sequential encoding and updating of event frequencies (Brunswik, 1939; Gallistel, 1990; Hume, 1739/1951; Shanks, 1991). For instance, research on foraging behavior indicates that bumblebees, ducks, rats, and ants behave as if they were good intuitive statisticians, highly sensitive to changes in frequency distributions in their environments (Gallistel, 1990; Real, 1991; Real \& Caraco, 1986). Similarly, research on frequency processing in humans indicates that humans, too, are sensitive to frequencies of various kinds, including frequencies of words, single letters, and letter pairs (e.g., Barsalou \& Ross, 1986; Hasher \& Zacks, 1979; Hintzman, 1976; Sedlmeier, Hertwig, \& Gigerenzer, 1995).

The sequential acquisition of information by updating event frequencies without artificially fixing the marginal frequencies (e.g., of disease and no-disease cases) is what we refer to as natural sampling (Kleiter, 1994). Brunswik's (1955) "representative sampling" is a special case of natural sampling. In contrast, in experimental research the marginal frequencies are typically fixed a priori. For instance, an experimenter may want to investigate 100 people with disease and a control group of 100 people without disease. This kind of sampling with fixed marginal frequencies is not what we refer to as natural sampling.

The evolutionary argument that cognitive algorithms were designed for frequency information, acquired through natural sampling, has implications for the computations an organism needs to perform when making Bayesian inferences. Here is the question to be answered: Assume an organism acquires information about the structure of its environment by the natural sampling of frequencies. What computations would the organism need to perform to draw inferences the Bayesian way?

Imagine an old, experienced physician in an illiterate society. She has no books or statistical surveys and therefore must rely solely on her experience. Her people have been afflicted by a previously unknown and severe disease. Fortunately, the physician has discovered a symptom that signals the disease, although not with certainty. In her lifetime, she has seen 1,000 


\section{Natural Sampling}

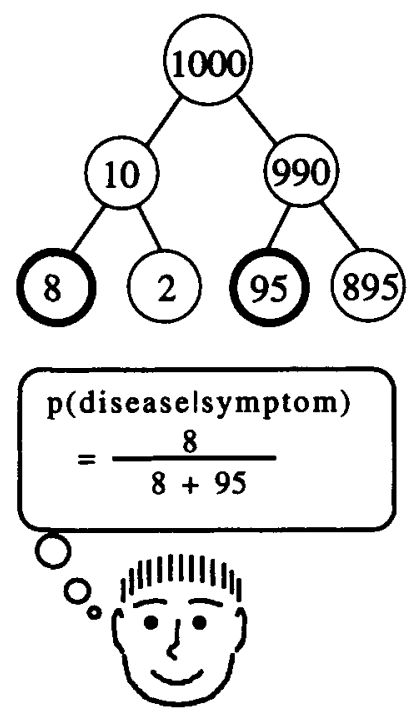

Standard Probability
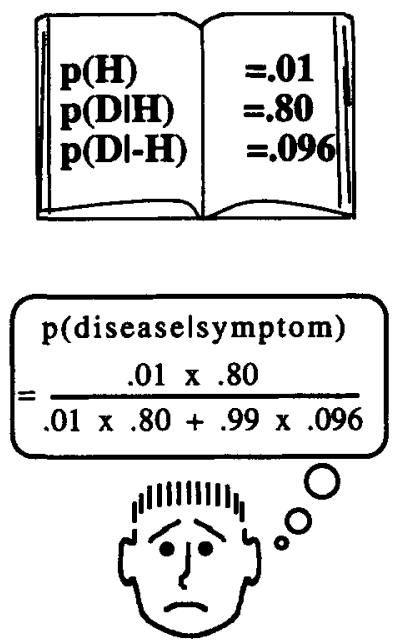

Figure 1. Bayesian inference and information representation (natural sampling of frequencies and standard probability format ).

people, 10 of whom had the disease. Of those 10,8 showed the symptom; of the 990 not afflicted, 95 did. Now a new patient appears. He has the symptom. What is the probability that he actually has the disease?

The physician in the illiterate society does not need a pocket calculator to estimate the Bayesian posterior. All she needs is the number of cases that had both the symptom and the disease (here, 8 ) and the number of symptom cases (here, $8+95$ ). A Bayesian algorithm for computing the posterior probability $p(H \mid D)$ from the frequency format (see Figure 1, left side) requires solving the following equation:

$$
p(H \mid D)=\frac{d \& h}{d \& h+d \&-h}=\frac{8}{8+95},
$$

where $d \& h$ ( $d$ ata and hypothesis) is the number of cases with symptom and disease, and $d \&-h$ is the number of cases having the symptom but lacking the disease. The physician does not need to keep track of the base rate of the disease. Her modern counterpart, the medical student who struggles with singleevent probabilities presented in medical textbooks, may on the other hand have to rely on a calculator and end up with little understanding of the result (see Figure 1, right side). ${ }^{1}$ Henceforth, when we use the term frequency format, we always refer to frequencies as defined by the natural sampling tree in Figure 1.

Comparison of Equations 1 and 2 leads to our first theoretical result:

Result 1: Computational demands. Bayesian algorithms are computationally simpler when information is encoded in a frequency format rather than a standard probability format. By "computationally simpler" we mean that (a) fewer operations (multiplication, addition, or division) need to be performed in Equation 2 than Equation 1, and (b) the operations can be per-

formed on natural numbers (absolute frequencies) rather than fractions (such as percentages).

Equations 1 and 2 are mathematically equivalent formulations of Bayes' theorem. Both produce the same result, $p(H \mid D)$ $=.078$. Equation 1 is a standard version of Bayes' theorem in today's textbooks in the social sciences, whereas Equation 2 corresponds to Thomas Bayes' (1763) original "Proposition 5" (see Earman, 1992).

Equation 2 implies three further (not independent) theoretical results concerning the estimation of a Bayesian posterior probability $p(H \mid D)$ in frequency formats (Kleiter, 1994).

Result 2: Attentional demands. Only two kinds of information need to be attended to in natural sampling: the absolute frequencies $d \& h$ and $d \&-h$ (or, alternately, $d \& h$ and $d$, where $d$ is the sum of the two frequencies). An organism does not need to keep track of the whole tree in Figure 1, but only of the two pieces of information contained in the bold circles. These are the hit and false alarm frequencies (not to be confused with hit and false alarm rates).

Result 3: Base rates need not be attended to. Neglect of base rates is perfectly rational in natural sampling. For instance, our physician does not need to pay attention to the base rate of the disease ( 10 out of 1,000; see Figure 1).

Result 4: Posterior distributions can be computed. Absolute frequencies can carry more information than probabilities. Information about the sample size allows inference beyond singlepoint estimates, such as the computation of posterior distributions, confidence intervals for posterior probabilities, and second-order probabilities (Kleiter, 1994; Sahlin, 1993). In this article, however, we focus only on single-point estimation.

For the design of the experiments reported below, it is important to note that the Bayesian algorithms (Equations 1 and 2) work on the final tally of frequencies (see Figure 1), not on the sequential record of updated frequencies. Thus, the same four results still hold even if nothing but the final tally is presented to the participants in an experiment.

\section{Information Format and Menu}

We propose to distinguish two aspects of information representation, information format and information menu. The standard probability format has a probability format, whereas a frequency format is obtained by natural sampling. However, as the second result (attentional demands) shows, there is another difference. The standard probability format displays three pieces of information, whereas two are sufficient in natural sampling. We use the term information menu to refer to the manner in which information is segmented into pieces within any format. The standard probability format displays the three pieces $p(H), p(D \mid H)$, and $p(D \mid-H)$ (often called base rate, hit rate, and false alarm rate, respectively). We refer to this as the standard menu. Natural sampling yields a more parsimonious menu with only two pieces of information, $d \& h$ and $d \&-h$ (or alternatively, $d \& h$ and $d$ ). We call this the short menu.

\footnotetext{
${ }^{1}$ This clinical example illustrates that the standard probability format is a convention rather than a necessity. Clinical studies often collect data that have the structure of frequency trees as in Figure 1. Such information can always be represented in frequencies as well as probabilities.
} 
Table 1

Information Formats and Menus for the Mammography Problem

Format and menu

Standard probability format

Standard frequency format

Short probability format

Short frequency format
Description of problem

The probability of breast cancer is $1 \%$ for women at age forty who participate in routine screening.

If a woman has breast cancer, the probability is $80 \%$ that she will get a positive mammography.

If a woman does not have breast cancer, the probability is $9.6 \%$ that she will also get a positive mammography.

A woman in this age group had a positive mammography in a routine screening. What is the probability that she actually has breast cancer? $\%$

10 out of every 1,000 women at age forty who participate in routine screening have breast cancer.

8 of every 10 women with breast cancer will get a positive mammography.

95 out of every 990 women without breast cancer will also get a positive mammography.

Here is a new representative sample of women at age forty who got a positive mammography in routine screening. How many of these women do you expect to actually have breast cancer? out of

The probability that a woman at age forty will get a positive mammography in routine screening is $10.3 \%$.

The probability of breast cancer and a positive mammography is $0.8 \%$ for a woman at age forty who participates in routine screening.

A woman in this age group had a positive mammography in a routine screening. What is the probability that she actually has breast cancer? \%

103 out of every 1,000 women at age forty get a positive mammography in routine screening.

8 out of every 1,000 women at age forty who participate in routine screening have breast cancer and a positive mammography.

Here is a new representative sample of women at age forty who got a positive mammography in routine screening. How many of these women do you expect to actually have breast cancer? _ out of
So far we have introduced the probability format with a standard menu and the frequency format with a short menu. However, information formats and menus can be completely crossed. For instance, if we replace the probabilities in the standard probability format with frequencies, we get a standard menu with a frequency format, or the standard frequency format. Table 1 uses the mammography problem to illustrate the four versions that result from crossing the two menus with the two formats. All four displays are mathematically equivalent in the sense that they lead to the same Bayesian posterior probability. In general, within the same format information can be divided into various menus; within the same menu, it can be represented in a range of formats.

To transform the standard probability format into the standard frequency format, we simply replaced $1 \%$ with " 10 out of 1,000 ," "80\%" with " 8 out of 10 ," and so on (following the tree in Figure 1) and phrased the task in terms of a frequency estimate. All else went unchanged. Note that whether the frequency format actually carries information about the sample size (e.g., that there were exactly 1,000 women) or not (as in Table 1, where it is said "in every 1,000 women") makes no difference for Results 1 to 3 because these relate to single-point estimates only (unlike Result 4).

What are the Bayesian algorithms needed to draw inferences from the two new format-menu combinations? The complete crossing of formats and menus leads to two important results. A Bayesian algorithm for the short probability format, that is, the probability format with a short menu (as in Table 1), amounts to solving the following equation:

$$
p(H \mid D)=\frac{p(D \& H)}{p(D)}
$$

This version of Bayes' theorem is equivalent to Equation 1. The algorithm for computing $p(H \mid D)$ from Equation 3, however, is computationally simpler than the algorithm for computing $p(H \mid D)$ from Equation 1.
What Bayesian computations are needed for the standard frequency format? Equation 2 specifies the computations for both the standard and short menus in frequency formats. The same algorithm is sufficient for both menus. In the standard frequency format of the mammography problem, for instance, the expected number of actual breast cancer cases among positive tests is computed as $8 /(8+95)$. Thus, we have the following two important theoretical results concerning formats (probability vs. frequency) and menus (standard vs. short):

Result 5: With a probability format, the Bayesian computations are simpler in the short menu than in the standard menu.

Result 6: With a frequency format, the Bayesian computations are the same for the two menus.

If the two pieces of information in the short menu are $d \& h$ and $d$, as in Table 1 , rather than $d \& h$ and $d \&-h$, then the Bayesian computations are even simpler because the sum in the denominator is already computed.

\section{Relative Frequencies}

Several studies of Bayesian inference have used standard probability formats in which one, two, or all three pieces of information were presented as relative frequencies rather than as single-event probabilities-although the task still was to estimate a single-event probability (e.g., Tversky \& Kahneman's, 1982 , cab problem). For instance, in the following version of the mammography problem, all information is represented in relative frequencies (in \%).

Relative frequency version (standard menu)

$1 \%$ of women at age forty who participate in routine screening have breast cancer. $80 \%$ of women with breast cancer will get positive mammographies. $9.6 \%$ of women without breast cancer will also get positive mammographies. A woman in this age group had a positive mammography in a routine screening. What is the probability that she actually has breast cancer? $\%$

Is the algorithm needed for relative frequencies computation- 
ally equivalent to the algorithm for frequencies, or to that for probabilities? The relative frequency format does not display the absolute frequencies needed for Equation 2. Rather, the numbers are the same as in the probability format, making the Bayesian computation the same as in Equation 1. This yields the following result:

Result 7: Algorithms for relative frequency versions are computationally equivalent to those for the standard probability format.

We tested several implications of Results 1 through 7 (except Result 4) in the studies reported below.

\section{The Format of the Single-Point Estimate}

Whether estimates relate to single events or frequencies has been a central issue within probability theory and statistics since the decline of the classical interpretation of probability in the $1830 \mathrm{~s}$ and $1840 \mathrm{~s}$. The question has polarized subjectivists and frequentists, additionally subdividing frequentists into moderate frequentists, such as R. A. Fisher (1955), and strong frequentists, such as J. Neyman (Gigerenzer et al., 1989). A single-point estimate can be interpreted as a probability or a frequency. For instance, clinical inference can be about the probability that a particular person has cancer or about the frequency of cancer in a new sample of people. Foraging (Simon, 1956; Stephens \& Krebs, 1986) provides an excellent example of a single-point estimate reasonably being interpreted as a frequency. The foraging organism is interested in making inferences that lead to satisfying results in the long run. Will it more often find food if it follows Cue $X$ or Cue $Y$ ? Here the singlepoint estimate can be interpreted as an expected frequency for a new sample. In the experimental research of the past two decades, participants were almost always required to estimate a single-event probability. But this need not be. In the experiments reported below, we asked people both for single-event probability and frequency estimates.

To summarize, mathematically equivalent information need not be computationally and psychologically equivalent. We have shown that Bayesian algorithms can depend on information format and menu, and we derived several specific results for when algorithms are computationally equivalent and when they are not.

\section{Cognitive Algorithms for Bayesian Inference}

How might the mind draw inferences that follow Bayes' theorem? Surprisingly, this question seems rarely to have been posed. Psychological explanations typically were directed at "irrational" deviations between human inference and the laws of probability; the "rational" seems not to have demanded an explanation in terms of cognitive processes. The cognitive account of probabilistic reasoning by Piaget and Inhelder (1951/ 1975 ), as one example, stops at the precise moment the adolescent turns "rational," that is, reaches the level of formal operations.

We propose three classes of cognitive algorithm for Bayesian inference: first, the algorithms corresponding to Equations 1 through 3; second, pictorial or graphical analogs of Bayes' theorem, as anticipated by Bayes' (1763) billiard table; and third, shortcuts that simplify the Bayesian computations in Equations 1 through 3.

\section{Pictorial Analogs}

We illustrate pictorial analogs and shortcut algorithms by drawing on actual performance from the studies reported below, in which none of the participants was familiar with Bayes' theorem. The German measles problem (in standard probability format and with the numerical information given in Study 2 ) serves as our example.

German measles during early pregnancy can cause severe prenatal damage in the child. Therefore, pregnant women are routinely tested for German measles infection. In one such test, a pregnant woman is found to be infected. In order best to advise this woman what to do, the physician first wants to determine the probability of severe prenatal damage in the child if a mother has German measles during early pregnancy. The physician has the following information: The probability of severe prenatal damage in a child is $0.5 \%$. The probability that a mother had German measles during early pregnancy if her child has severe prenatal damage is $40 \%$. The probability that a mother had German measles during early pregnancy if her child does not have severe prenatal damage is $0.01 \%$. What is the probability of severe prenatal damage in the child if the mother has German measles during early pregnancy? $\%$

The "beam analysis" (see Figure 2) is a pictorial analog of Bayes' theorem developed by one of our research participants. This individual represented the class of all possible outcomes (child has severe prenatal damage and child does not have severe prenatal damage) by a beam. He drew inferences (here, about the probability that the child has severe prenatal damage) by cutting off two pieces from each end of the beam and comparing their size. His algorithm was as follows:

Step 1: Base rate cut. Cut off a piece the size of the base rate from the right end of the beam.

Step 2: Hit rate cut. From the right part of the beam (base rate piece), cut off a proportion $p(D \mid H)$.

Step 3: False alarm cut. From the left part of the beam, cut off a proportion $p(D \mid-H)$.

Step 4: Comparison. The ratio of the right piece to both pieces is the posterior probability.

This algorithm amounts to Bayes' theorem in the form of Equation 1.

\section{Shortcut Algorithms: Probability Format}

We have observed in our experiments three elementary shortcuts and several combinations thereof. For instance, by ignoring small "slices," one can simplify the computation without much loss of accuracy, which is easily compensated for by the fact that less computation means a reduced chance of computational errors. We illustrate these shortcuts using the beam analysis (see Figure 2). However, these shortcuts are not restricted to pictorial analogs, and they were used by many of our participants.

\section{Rare-Event Shortcut}

Rare events-that is, outcomes with small base rates, such as severe prenatal damage-enable simplification of the Bayesian 


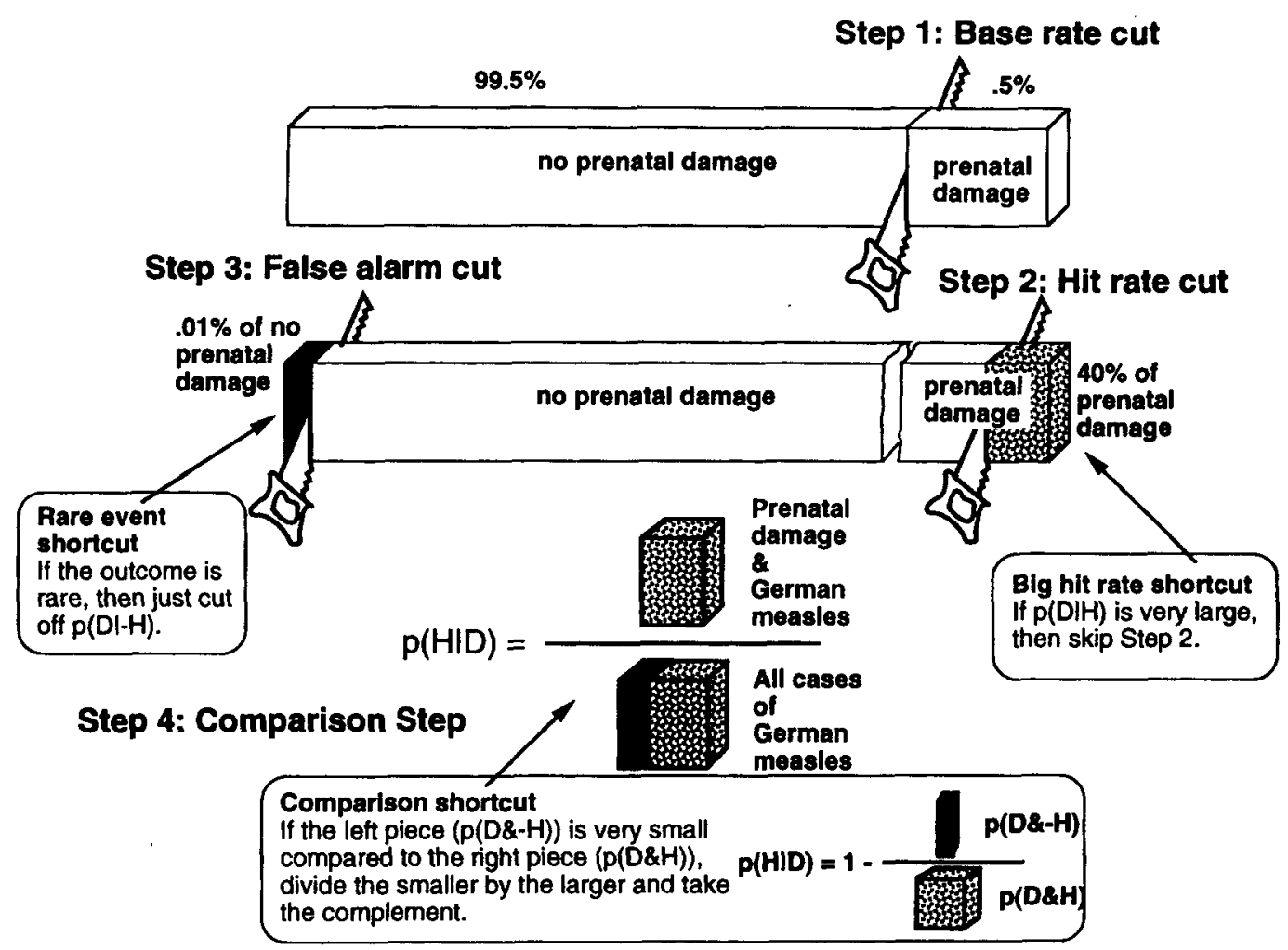

Figure 2. A Bayesian algorithm invented by one of our research participants. The "beam cut" is illustrated for the German measles problem in the standard probability format. $H$ stands for "severe prenatal damage in the child," and $D$ stands for "mother had German measles in early pregnancy." The information is $p(H)$ $=0.5 \%, p(D \mid H)=40 \%$, and $p(D \mid-H)=0.01 \%$. The task is to infer $p(H \mid D)$.

inference with little reduction in accuracy. If an event is rare, that is, if $p(H)$ is very small, and $p(-H)$ is therefore close to 1.0 , then $p(D \mid-H) p(-H)$ can be approximated by $p(D \mid-H)$. That is, instead of cutting the proportion $p(D \mid-H)$ of the left part of the beam (Step 3), it is sufficient to cut a piece of absolute size $p(D \mid-H)$. The rare-event shortcut (see Figure 2 ) is as follows:

\section{IF the event is rare,}

THEN simplify Step 3: Cut a piece of absolute size $p(D \mid-H)$.

This shortcut corresponds to the approximation

$$
p(H \mid D) \approx p(H) p(D \mid H) /[p(H) p(D \mid H)+p(D \mid-H)] .
$$

The shortcut works well for the German measles problem, where the base rate of severe prenatal damage is very small, $p(H)=.005$. The shortcut estimates $p(H \mid D)$ as .9524 , whereas Bayes' theorem gives .9526. It also works with the mammography problem, where it generates an estimate of .077 , compared with .078 from Bayes' theorem.

\section{Big Hit-Rate Shortcut}

Large values of $p(D \mid H)$ (such as high diagnosticities in medical tests; that is, excellent hit rates) allow one to skip Step 2 with little loss of accuracy. If $p(D \mid H)$ is very large, then the
$p(H)$ piece is practically the same size as the piece one obtains from cutting all but a tiny sliver from the $p(H)$ piece. The big hit-rate shortcut is then as follows:

IF $p(D \mid H)$ is very large,

\section{THEN skip Step 2.}

This shortcut corresponds to the approximation

$$
p(H \mid D) \approx p(H) /[p(H)+p(-H) p(D \mid-H)] .
$$

The big hit-rate shortcut would not work as well as the rareevent shortcut in the German measies problem because $p(D \mid H)$ is only .40 . Nevertheless, the shortcut estimate is only a few percentage points removed from that obtained with Bayes' theorem ( .980 instead of .953 ). The big hit-rate shortcut works well, to offer one instance, in medical diagnosis tasks where the hit rate of a test is high (e.g., around .99 as in HIV tests).

\section{Comparison Shortcut}

If one of the two pieces obtained in Steps 2 and 3 is small relative to the other, then the comparison in Step 4 can be simplified with little loss of accuracy. For example, German measles in early pregnancy and severe prenatal damage in the child occur more frequently than do German measles and no severe damage. More generally, if $D \& H$ cases are much more frequent 
than $D \&-H$ cases (as in the German measles problem), or vice versa (as in the mammography problem), then only two pieces (rather than three) need to be related in Step 4. The comparison shortcuts for these two cases are as follows:

\section{IF D\&-H occurs much more often than $D \& H$,}

THEN simplify Step 4: Take the ratio of D\&H(right piece)

$$
\text { to } D \&-H \text { (left piece) as the posterior probability. }
$$

This shortcut corresponds to the approximation

$$
p(H \mid D) \approx p(H) p(D \mid H) / p(-H) p(D \mid-H) .
$$

Note that the right side of this approximation is equivalent to the posterior odds ratio $p(H \mid D) / p(-H \mid D)$. Thus, the comparison shortcut estimates the posterior probability by the posterior odds ratio.

IF D\&H occurs much more often than $D \&-H$,

THEN simplify Step 4: Take the ratio of $D \&-H$ (left piece)

to $D \& H$ (right piece) as the complement

of the posterior probability.

This shortcut corresponds to the approximation

$$
p(H \mid D) \approx 1-p(-H) p(D \mid-H) / p(H) p(D \mid H) .
$$

The comparison shortcut estimates $p(H \mid D)$ as .950 in the German measles problem, whereas Bayes' theorem gives .953. The comparison shortcut is simpler when the $D \&-H$ cases are the more frequent ones, which is typical for medical diagnosis, where the number of false alarms is much larger than the number of hits, as in mammography and HIV tests.

\section{Multiple Shortcuts}

Two or three shortcuts can be combined, which results in a large computational simplification. What we call the quick-andclean shortcut combines all three. Its conditions include a rare event, a large hit rate, and many $D \&-H$ cases compared with $D \& H$ cases (or vice versa). The quick-and-clean shortcut is as follows:

IF an event $H$ is rare, $p(D / H)$ high, and $D \&-H$

cases much more frequent than $D \& H$ cases,

THEN simply divide the base rate by the false alarm rate.

This shortcut corresponds to the approximation

$$
p(H \mid D) \approx p(H) / p(D \mid-H) .
$$

The conditions of the quick-and-clean shortcut seem to be not infrequently satisfied. Consider routine HIV testing: According to present law, the U.S. immigration office makes an HIV test a condition sine qua non for obtaining a green card. Mr. Quick has applied for a green card and wonders what a positive test result indicates. The information available is a base rate of .002 , a hit rate of .99 , and a false alarm rate of .02; all three conditions for the quick-and-clean shortcut are thus satisfied. Mr. Quick computes $.002 / .02=.10$ as an estimate of the posterior probability of actually being infected with the HIV virus if he tests positive. Bayes' theorem results in .09 . The shortcut is therefore an excellent approximation. Alternately, if $D \& H$ cases are more frequent, then the quick-and-clean shortcut is to divide the false alarm rate by the base rate and to use this as an estimate for 1 $-p(H \mid D)$. In the mammography and German measles problems, where the conditions are only partially satisfied, the quickand-clean shortcut still leads to surprisingly good approximations. The posterior probability of breast cancer is estimated at $.01 / .096$, which is about .10 (compared with .078 ), and the posterior probability of severe prenatal damage is estimated as .98 (compared with .953).

\section{Shortcuts: Frequency Format}

Does the standard frequency format invite the same shortcuts? Consider the inference about breast cancer from a positive mammography, as illustrated in Figure 1. Would the rare-event shortcut facilitate the Bayesian computations? In the probability format, the rare-event shortcut uses $p(D \mid-H)$ to approximate $p(-H) p(D \mid-H)$; in the frequency format, the latter corresponds to the absolute frequency $95(d \&-h)$ and no approximation is needed. Thus, a rare-event shortcut is of no use and would not simplify the Bayesian computation in frequency formats. The same can be shown for the big hit-rate shortcut for the same reason. The comparison shortcut, however, can be applied in the frequency format:

IF $d \&-h$ occurs much more often than $d \& h$, THEN compute $d \& h / d \&-h$.

The condition and the rationale are the same as in the probability format.

To summarize, we proposed three classes of cognitive algorithms underlying Bayesian inference: (a) algorithms that satisfy Equations 1 through 3; (b) pictorial analogs that work with operations such as "cutting" instead of multiplying (Figure 2); and (c) three shortcuts that approximate Bayesian inference well when certain conditions hold.

\section{Predictions}

We now derive several predictions from the theoretical results obtained. The predictions specify conditions that do and do not make people reason the Bayesian way. The predictions should hold independently of whether the cognitive algorithms follow Equations 1 through 3, whether they are pictorial analogs of Bayes' theorem, or whether they include shortcuts.

Prediction 1: Frequency formats elicit a substantially higher proportion of Bayesian algorithms than probability formats. This prediction is derived from Result 1, which states that the Bayesian algorithm is computationally simpler in frequency formats. $^{2}$

\footnotetext{
${ }^{2}$ At the point when we introduced Result 1 , we had dealt solely with the standard probability format and the short frequency format. However, Prediction 1 also holds when we compare formats across both menus. This is the case because (a) the short menu is computationally simpler in the frequency than in the probability format, because the frequency format involves calculations with natural numbers and the probability format with fractions, and (b) with a frequency format, the Bayesian computations are the same for the two menus (Result 6).
} 
Prediction 2: Probability formats elicit a larger proportion of Bayesian algorithms for the short menu than for the standard menu. This prediction is deduced from Result 5 , which states that with a probability format, the Bayesian computations are simpler in the short menu than in the standard menu.

Prediction 3: Frequency formats elicit the same proportion of Bayesian algorithms for the two menus. This prediction is derived from Result 6, which states that with a frequency format, the Bayesian computations are the same for the two menus.

Prediction 4: Relative frequency formats elicit the same (small) proportion of Bayesian algorithms as probability formats. This prediction is derived from Result 7, which states that the Bayesian algorithms are computationally equivalent in both formats.

\section{Operational Criteria for Identifying Cognitive Algorithms}

The data we obtained for each of several thousand problem solutions were composed of a participant's (a) probability or frequency estimate and (b) on-line protocol ("write aloud" protocol) of his or her reasoning. Data type (a) allowed for an outcome analysis, as used exclusively in most earlier studies on Bayesian inference, whereas data type (b) allowed additionally for a process analysis.

\section{Double Check: Outcome and Process}

We classified an inferential process as a Bayesian algorithm only if (a) the estimated probability or frequency was exactly the same as the value calculated from applying Bayes' theorem to the information given (outcome criterion), and (b) the online protocol specified that one of the Bayesian computations defined by Equations 1 through 3 or one (or several) of the Bayesian shortcut algorithms was used, either by means of calculation or pictorial representation (process criterion). We applied the same strict criteria to identify non-Bayesian cognitive algorithms.

\section{Outcome: Strict Rounding Criterion}

By the phrase "exactly the same" in the outcome criterion, we mean the exact probability or frequency, with exceptions made for rounding up or down to the next full percentage point (e.g., in the German measles problem, where rounding the probability of $95.3 \%$ down or up to a full percentage point results in $95 \%$ or $96 \%$ ). If, for example, the on-line protocol showed that a participant in the German measles problem had used the rare-event shortcut and the answer was $95 \%$ or $96 \%$ (by rounding), this inferential process was classified as a Bayesian algorithm. Estimates below or above were not classified as Bayesian algorithms: If, for example, another participant in the same problem used the big hit-rate shortcut (where the condition for this shortcut is not optimally satisfied) and accordingly estimated $98 \%$, this was not classified as a Bayesian algorithm. Cases of the latter type ended up in the category of "less frequent algorithms." This example illustrates the strictness of the joint criteria. The strict rounding criterion was applied to the frequency format in the same way as to the probability format.

When a participant answered with a fraction-such as that resulting from Equation 3-without performing the division, this was treated as if she or he had performed the division. We did not want to evaluate basic arithmetic skills. Similarly, if a participant arrived at a Bayesian equation but made a calculation error in the division, we ignored the calculation error.

\section{Process: "Write Aloud" Protocols}

Statistical reasoning often involves pictorial representations as well as computations. Neither are easily expressed verbally, as in "think aloud" methods. Pictorial representations and computations consequently are usually expressed by drawing and writing down equations and calculations. We designed a "write aloud" technique for tracking the reasoning process without asking the participant to talk aloud either during or after the task.

The "write aloud" method consisted of the following steps. First, participants were instructed to record their reasoning unless merely guessing the answer. We explained that a protocol may contain a variety of elements, such as diagrams, pictures, calculations, or whatever other tools one may use to find a solution. Each problem was on a separate page, which thus allowed ample space for notes, drawings, and calculations. Second, after a participant had completed a problem, he or she was asked to indicate whether the answer was based on a calculation or on a guess. Third, when a "write aloud" protocol was unreadable or the process that generated the probability estimate was unclear, and the participant had indicated that the given result was a calculation, then he or she was interviewed about the particular problem after completing all tasks. This happened only a few times. If a participant could not immediately identify what his or her notes meant, we did not inquire further.

The "write aloud" method avoids two problems associated with retrospective verbal reports: that memory of the cognitive algorithms used may have faded by the time of a retrospective report (Ericsson \& Simon, 1984) and that participants may have reported how they believe they ought to have thought rather than how they actually thought (Nisbett \& Wilson, 1977).

We used the twin criteria of outcome and process to crosscheck outcome by process and vice versa. The outcome criterion prevents a shortcut algorithm from being classified as a Bayesian algorithm when the precondition for the shortcut is not optimally satisfied. The process criterion protects against the opposite error, that of inferring from a probability judgment that a person actually used a Bayesian algorithm when he or she did not.

We designed two studies to identify the cognitive algorithms and test the predictions. Study 1 was designed to test Predictions 1,2 , and 3 .

\section{Study 1: Information Formats and Menus}

\section{Method}

\section{Participants}

Sixty students, 21 men and 39 women from 10 disciplines (predominantly psychology) from the University of Salzburg, Austria, were paid for their participation. The median age was 21 years. None of the participants was familiar with Bayes' theorem. 
Table 2

Information Given and Bayesian Solutions for the 15 Problems in Study 1

\begin{tabular}{|c|c|c|c|c|c|c|c|c|}
\hline \multicolumn{2}{|c|}{ Task: Estimate $p(H \mid D)$} & \multicolumn{6}{|c|}{ Information (standard frequency format) } & \multirow{2}{*}{$\frac{\text { Bayes }^{b}}{p(H \mid D)}$} \\
\hline $\boldsymbol{H}$ & $D$ & & $H$ & & & & $\boldsymbol{H}$ & \\
\hline Breast cancer & Mammography positive & 10 & 1,000 & 8 & 10 & 95 & 990 & 7.77 \\
\hline Prenatal damage in child & German measles in mother & 21 & 10,000 & 10 & 21 & 50 & 10,000 & 16.70 \\
\hline Blue cab & Eyewitness says "blue" & 15 & 100 & 12 & 15 & 17 & 85 & 41.38 \\
\hline AIDS & HIV test positive & 100 & $1,000,000$ & 100 & 100 & 1,000 & $1,000,000$ & 9.09 \\
\hline Heroin addict & Fresh needle prick & 10 & 100,000 & 10 & 10 & 190 & 100,000 & 5.00 \\
\hline Pregnant & Pregnancy test positive & 20 & 1,000 & 19 & 20 & 5 & 980 & 79.17 \\
\hline Car accident & Driver drunk & 100 & 10,000 & 55 & 100 & 500 & 9,900 & 9.91 \\
\hline Bad posture in child & Heavy books carried daily & 50 & 1,000 & 20 & 50 & 190 & 950 & 9.52 \\
\hline Accident on way to school & Child lives in urban area & 30 & 1,000 & 27 & 30 & 388 & 970 & 6.51 \\
\hline Commiting suicide & Professor & 240 & $1,000,000$ & 36 & 240 & 120,000 & $1,000,000$ & 0.03 \\
\hline Red ball & Marked with star & 400 & 500 & 300 & 400 & 25 & 100 & 92.31 \\
\hline Choosing course in economics & Career oriented & 300 & 1,000 & 210 & 300 & 350 & 700 & 37.50 \\
\hline Active feminist & Bank teller & 5,000 & 100,000 & 20 & 5,000 & 2,000 & 95,000 & 0.99 \\
\hline Pimp & Wearing a Rolex & 50 & $1,000,000$ & 40 & 50 & 500 & $1,000,000$ & 7.41 \\
\hline Admission to school & Particular placement test result & 360 & 1,000 & 270 & 360 & 128 & 640 & 67.84 \\
\hline
\end{tabular}

- The representation of the information is shown only for the standard frequency format (frequency format and standard menu). The other representations (see Table 1) can be derived from this. The two numbers for each piece of information are connected by an "out of" relation; for example, the information concerning $H$ in the first problem should be read as " 10 out of 1,000 ." " Probabilities are expressed as percentages.

Particjpants were studied individually or in small groups of 2 or 3 (in two cases, 5). We informed participants that they would need approximately $\mathrm{l}$ hr for each session but that they could have more time if necessary. On the average, students worked $73 \mathrm{~min}$ in the first session (range $=25-180 \mathrm{~min}$ ) and $53 \mathrm{~min}$ in the second (range $=30-120 \mathrm{~min}$ ).

\section{Procedure}

We used two formats, probability and frequency, and two menus, standard and short. The two formats were crossed with the two menus, so four versions were constructed for each problem. There were 15 problems, including the mammography problem (Eddy, 1982; see Table 1), the cab problem (Tversky \& Kahneman, 1982), and a short version of Ajzen's (1977) economics problem. The four versions of each problem were constructed in the same way as explained before with the mammography problem (see Table 1 ). ${ }^{3}$ In the frequency format, participants were always asked to estimate the frequency of " $h$ out of $d$ "; in the probability format, they were always asked to estimate the probability $p(H \mid D)$. Table 2 shows for each of the 15 problems the information given in the standard frequency format; the information specified in the other three versions can be derived from that.

Participants were randomly assigned to two groups, with the members of both answering each of the 15 problems in two of the four versions. One group received the standard probability format and the short frequency format; the other, the standard frequency format and the short probability format. Each participant thus worked on 30 tasks. There were two sessions, 1 week apart, with 15 problems each. Formats and menus were distributed equally over the sessions. The two versions of one problem were always given in different sessions. The order of the problems was determined randomly, and two different random orders were used within each group.

\section{Results}

\section{Bayesian Algorithms}

Prediction 1: Frequency formats elicit a substantially higher proportion of Bayesian algorithms than probability formats. Do frequency formats foster Bayesian reasoning? Yes. Fre- quency formats elicited a substantially higher proportion of Bayesian algorithms than probability formats: $46 \%$ in the standard menu and $50 \%$ in the short menu. Probability formats, in contrast, elicited $16 \%$ and $28 \%$, for the standard menu and the short menu, respectively. These proportions of Bayesian algorithms were obtained by the strict joint criteria of process and outcome and held fairly stable across 15 different inference problems. Note that $50 \%$ Bayesian algorithms means $50 \%$ of all answers, and not just of those answers where a cognitive algorithm could be identified. The percentage of identifiable cognitive algorithms across all formats and menus was $84 \%$.

Figure 3 shows the proportions of Bayesian algorithms for each of the 15 problems. The individual problems mirror the general result. For each problem, the standard probability format elicited the smallest proportion of Bayesian algorithms. Across formats and menus, in every problem Bayesian algorithms were the most frequent.

The comparison shortcut was used quite aptly in the standard frequency format, that is, only when the precondition of the algorithm was satisfied to a high degree. It was most often used in the suicide problem, in which the ratio between $D \& H$ cases and $D \&-H$ cases was smallest (Table 2 ), that is, in which the precondition was best satisfied. Here, 9 out of 30 participants used the comparison shortcut (and 5 participants used the Bayesian algorithm without a shortcut). In all 20 instances where the shortcut was used, 17 satisfied the strict outcome criterion, and the remaining 3 were accurate to within 4 percentage points.

Because of the strict rounding criterion, the numerical esti-

\footnotetext{
${ }^{3}$ If the $Y$ number in " $X$ out of $Y$ " was large and odd, such as 9,950 , we rounded the number to a close, more simple number, such as 10,000 . The German measles problem is an example. This made practically no difference for the Bayesian calculation and was meant to prevent participants from being puzzled by odd $Y$ numbers.
} 


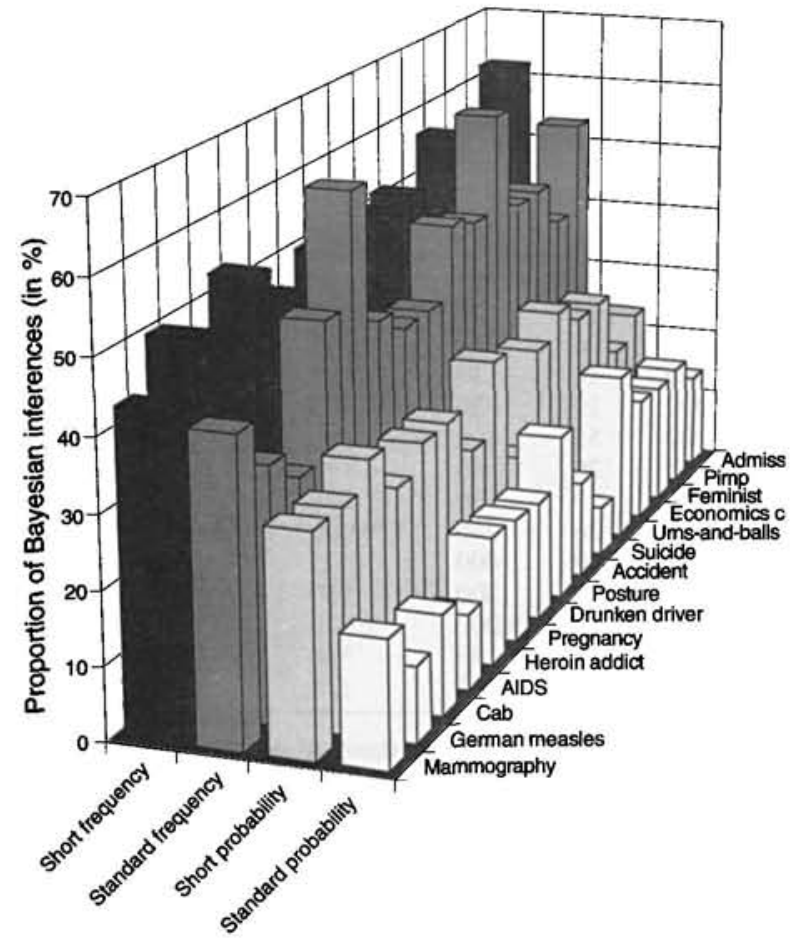

Figure 3. Proportion of Bayesian algorithms in the 15 problems of Study 1. "Standard probability" stands for probability format with standard menu, "short frequency" stands for frequency format with short menu, and so on.

mates of the participants using a Bayesian algorithm can be directly read from Table 2 . For instance, in the short frequency version of the mammography problem, $43.3 \%$ of participants (see Figure 3) came up with a frequency estimate of 8 out of 103 (or another value equivalent to $7.8 \%$, or within $7 \%$ and $8 \%$ ).

The empirical result in Figure 3 is consistent with the theoretical result that frequency formats can be handled by Bayesian algorithms that are computationally simpler than those required by probability formats.

Prediction 2: Probability formats elicit a larger proportion of Bayesian algorithms for the short menu than for the standard menu. The percentages of Bayesian algorithms in probability formats were $16 \%$ and $28 \%$ for the standard menu and the short menu, respectively. Prediction 2 holds for each of the 15 problems (Figure 3).

Prediction 3: The proportion of Bayesian algorithms elicited by the frequency format is independent of the menu. The effect of the menu largely, but not completely, disappeared in the frequency format. The short menu elicited 3.7 percentage points more Bayesian algorithms than the standard menu. The residual superiority of the short menu could have the following cause: Result 2 (attentional demands) states that in natural sampling it is sufficient for an organism to monitor either the frequencies $d \& h$ and $d$ or $d \& h$ and $d \&-h$. We have chosen the former pair for the short menus in our studies and thus reduced the Bayesian computation by one step, that of adding up $d \& h$ and $d \&-h$ to $d$, which was part of the Bayesian computation in the standard but not the short menu. This additional computational step is consistent with the small difference in the propor- tions of Bayesian algorithms found between the two menus in the frequency formats.

How does the impact of format on Bayesian reasoning compare with that of menu? The effect of the format was about three times larger than that of the menu (29.9 and 21.6 percentage points difference compared with 12.1 and 3.7 ). Equally striking, the largest percentage of Bayesian algorithms in the two probability menus $(28 \%)$ was considerably smaller than the smallest in the two frequency menus ( $46 \%)$.

\section{Non-Bayesian Algorithms}

We found three major non-Bayesian cognitive algorithms (see Table 3).

Joint occurrence. The most frequent non-Bayesian algorithm was a computation of the joint occurrence of $D$ and $H$. Depending on the menu, this involved calculating $p(H) p(D \mid H)$, or simply "picking" $p(H \& D)$ (or the corresponding values for the frequency format). Joint occurrence does not neglect base rates; it neglects the false alarm rate in the standard menu and $p(D)$ in the short menu. Joint occurrence always underestimates the Bayesian posterior unless $p(D)=1$. From participants' "write aloud" protocols, we learned about a variant, which we call adjusted joint occurrence, in which the participant starts with joint occurrence and adjusts it slightly ( 5 or fewer percentage points).

Fisherian. Not all statisticians are Bayesians. R. A. Fisher, who invented the analysis of variance and promoted significance testing, certainly was not. In Fisher's (1955) theory of significance testing, an inference from data $D$ to a null hypothesis $H_{o}$ is based solely on $p\left(D \mid H_{o}\right)$, which is known as the "exact level of significance." The exact level of significance ignores base rates and false alarm rates. With some reluctance, we labeled the second most frequent non-Bayesian algorithm-picking $p(D \mid H)$ and ignoring everything else-"Fisherian." Our hesitation lay in the fact that it is one thing to ignore everything else besides $p(D \mid H)$, as Fisher's significance testing method does, and quite another thing to confuse $p(D \mid H)$ with $p(H \mid D)$. For instance, a $p$ value of $1 \%$ is often erroneously believed to mean, by both researchers (Oakes, 1986) and some statistical textbook authors (Gigerenzer, 1993b), that the probability of the null hypothesis being true is $1 \%$. Thus the term Fisherian refers to this widespread misinterpretation rather than to Fisher's actual ideas (we hope that Sir Ronald would forgive us).

There exist several related accounts of the strategy for inferring $p(H \mid D)$ solely on the basis of $p(D \mid H)$. Included in these are the tendency to infer "cue validity" from "category validity" (Medin, Wattenmaker, \& Michalski, 1987) and the related thesis that people have spontaneous access to sample spaces that correspond to categories (e.g., cancer) rather than to features associated with categories (Gavanski \& Hui, 1992). Unlike the Bayesian algorithms and joint occurrence, the Fisherian algorithm is menu specific: It cannot be elicited from the short menu. We observed from participants' "write aloud" protocols the use of a variant, which we call adjusted Fisherian, in which the participant started with $p(D \mid H)$ and then adjusted this value slightly ( 5 or fewer percentage points) in the direction of some other information.

Likelihood subtraction. Jerzy Neyman and Egon S. Pearson challenged Fisher's null-hypothesis testing (Gigerenzer, 1993b). They argued that hypothesis testing is a decision between (at 
Table 3

Cognitive Algorithms in Study 1

\begin{tabular}{|c|c|c|c|c|c|c|c|}
\hline \multirow{3}{*}{$\begin{array}{l}\text { Cognitive } \\
\text { algorithm }\end{array}$} & \multirow[b]{3}{*}{ Formal equivalent } & \multicolumn{4}{|c|}{ Information format and menu } & \multirow[b]{3}{*}{ Total } & \multirow[b]{3}{*}{$\%$ of total } \\
\hline & & \multicolumn{2}{|c|}{ Probability } & \multicolumn{2}{|c|}{ Frequency } & & \\
\hline & & Standard & Short & Standard & Short & & \\
\hline Bayesian & $p(H \mid D)$ & 69 & 126 & 204 & 221 & 620 & 34.9 \\
\hline Joint occurrence & $p(H \& D)$ & 39 & 97 & 20 & 97 & 253 & 14.3 \\
\hline $\begin{array}{l}\text { Adjusted joint } \\
\text { occurrence }\end{array}$ & $n(H \& D)+05$ & & 64 & & 55 & 110 & 67 \\
\hline Fisherian & $p(D \mid H)$ & 67 & & 36 & (3) & 103 & $\begin{array}{l}0.7 \\
5.8\end{array}$ \\
\hline Adjusted Fisherian & $p(D \mid H) \pm .05$ & 32 & & 19 & & 51 & 2.9 \\
\hline Multiply all & $p(D) p(H \& D)$ & & 79 & & 12 & 91 & 5.1 \\
\hline $\begin{array}{l}\text { Likelihood } \\
\text { subtraction }\end{array}$ & $p(D \mid H)-p(D \mid-H)$ & 30 & & 4 & & 34 & 1.9 \\
\hline Base rate only & $p(H)$ & 6 & & 13 & & 19 & 1.1 \\
\hline $\begin{array}{l}\text { Less frequent } \\
\text { algorithms }\end{array}$ & & & & & & & \\
\hline$(<1 \%$ of total $)$ & & 71 & 32 & 60 & 29 & 192 & 10.8 \\
\hline Not identified & & 119 & 52 & 89 & 32 & 292 & 16.5 \\
\hline Total & & 433 & 450 & 445 & 446 & $1,774^{\mathrm{a}}$ & 100.0 \\
\hline
\end{tabular}

Note. Numbers are absolute frequencies.

The sum of total answers in Table 3 is 1,774 rather than 1,800 ( 60 participants times 30 tasks) because of some participants' refusals to answer and a few missing data.

least ) two hypotheses that is based on a comparison of the probability of the observed data under both, which they construed as the likelihood ratio $p(D \mid H) / p(D \mid-H)$. We observed a simplistic version of the Neyman-Pearson method, the likelihood subtraction algorithm, which computes $p(D \mid H)-p(D \mid-H)$. As in Neyman-Pearson hypotheses testing, this algorithm makes no use of prior probabilities and thus neglects base rate information. The cognitive algorithm is menu specific (it can only be elicited by the standard menu) and occurred predominantly in the probability format. On Robert Nozick's account, likelihood subtraction is said to be a measure of evidential support (see Schum, 1994), and McKenzie (1994) has simulated the performance of this and other non-Bayesian algorithms.

Others. There were cases of multiply all in the short menu (the logic of which escaped us) and a few cases of base rate only in the standard menu (a proportion similar to that reported in Gigerenzer, Hell, \& Blank, 1988). We identified a total of $10.8 \%$ other algorithms; these are not described here because each was used in fewer than $1 \%$ of the solutions.

\section{Summary of Study 1}

The standard probability format-the information representation used in most earlier studies-elicited $16 \%$ Bayesian algorithms. When information was presented in a frequency format, this proportion jumped to $46 \%$ in the standard menu and $50 \%$ in the short menu. The results of Study 1 are consistent with Predictions 1, 2, and 3. Frequency formats, in contrast to probability formats, "invite" Bayesian algorithms, a result that is consistent with the computational simplicity of Bayesian algorithms entailed by frequencies. Two of the three major classes of non-Bayesian algorithms our participants used-Fisherian and likelihood subtraction-mimic statistical inferential algorithms used and discussed in the literature.

\section{Study 2: Cognitive Algorithms for Probability Formats}

In this study we concentrated on probability and relative frequency rather than on frequency formats. Thus, in this study, we explored cognitive algorithms in the two formats used by almost all previous studies on base rate neglect. Our goal was to test Prediction 4 and to provide another test of Prediction 2.

We used two formats, probability and relative frequency, and three menus: standard, short, and hybrid. The hybrid menu displayed $p(H), p(D \mid H)$, and $p(D)$, or the respective relative frequencies. The first two pieces come from the standard menu, the third from the short menu. With the probability format and the hybrid menu, a Bayesian algorithm amounts to solving the following equation:

$$
p(H \mid D)=\frac{p(H) p(D \mid H)}{p(D)}
$$

The two formats and the three menus were mathematically interchangeable and always entailed the same posterior probability. However, the Bayesian algorithm for the short menu is computationally simpler than that for the standard menu, and the hybrid menu is in between; therefore the proportion of Bayesian algorithms should increase from the standard to the hybrid to the short menu (extended Prediction 2). In contrast, the Bayesian algorithms for the probability and relative frequency formats are computationally equivalent; therefore there should be no difference between these two formats (Prediction 4). 


\section{Method}

\section{Participants}

Fifteen students from the fields of biology, linguistics, English studies, German studies, philosophy, political science, and management at the University of Konstanz, Germany, served as participants. Eight were men, and 7 were women; the median age was 22 years. They were paid for their participation and studied in one group. None was familiar with Bayes' theorem.

\section{Procedure}

We used 24 problems, half from Study 1 and the other half new. ${ }^{4}$ For each of the 24 problems, the information was presented in three menus, which resulted in a total of 72 tasks. Each participant performed all 72 tasks. We randomly assigned half of the problems to the probability format and half to the relative frequency format; each participant thus answered half of the problems in each format. All probabilities and relative frequencies were stated in percentages. The questions were always posed in terms of single-event probabilities.

Six 1-hr sessions were scheduled, spaced equally over a 3-week interval. In each session, 12 tasks were performed. Participants received the 72 tasks in different orders, which were determined as follows: (a) Tasks that differed only in menu were never given in the same session, and (b) the three menus were equally frequent in every session. Within these two constraints, the 72 tasks were randomly assigned to six groups of 12 tasks each, with the 12 tasks within each group randomly ordered. These six groups were randomly assigned to the six sessions for each participant. Finally, to control for possible order effects within the three (two) pieces of information (Kroznick, Li, \& Lehman, 1990), we determined the order randomly for each participant.

The procedure was the same as in Study 1, except that we had participants do an even larger number of inference problems and that we did not use the "write aloud" instruction. However, participants could (and did) spontaneously "write aloud." After a student had completed all 72 tasks, he or she received a new booklet. This contained copies of a sample of 6 tasks the student had worked on, showing the student's probability estimates, notes, drawings, calculations, and so forth. Attached to each task was a questionnaire in which the student was asked, "Which information did you use for your estimates?" and "How did you derive your estimate from the information? Please describe this process as precisely as you can." Thus, in Study 2, we had only limited "write aloud" protocols and after-the-fact interviews available. A special prize of 25 deutsche marks was offered for the person with the best performance.

\section{Results}

We could identify cognitive algorithms in $67 \%$ of 1,080 probability judgments. Table 4 shows the distribution of the cognitive algorithms for the two formats as well as for the three menus.

\section{Bayesian Algorithms}

Prediction 4: Relative frequency formats elicit the same (small) proportion of Bayesian algorithms as probability formats. Table 4 shows that the number of Bayesian algorithms is not larger for the relative frequency format (60) than for the probability format (66). Consistent with Prediction 4, the numbers are about the same. More generally, Bayesian and nonBayesian algorithms were spread about equally between the two formats. Therefore, we do not distinguish probability and relative frequency formats in our further analysis.

Prediction 2 (extended to three menus): The proportion of
Bayesian algorithms elicited by the probability format is lowest for the standard menu, followed in ascending order by the hybrid and short menus. Study 2 allows for a second test of Prediction 2 , now with three menus. Bayesian algorithms almost doubled from the standard to the hybrid menu and almost tripled in the short menu (Table 4 ). Thus the prediction holds again. In Study 1, the standard probability menu elicited $16 \%$ Bayesian algorithms, as opposed to $28 \%$ for the short menu. In Study 2, the corresponding percentages of Bayesian algorithms in probability formats were generally lower, $6.4 \%$ and $17.5 \%$. What remained unchanged, however, was the difference between the two menus, about 12 percentage points, which is consistent with Prediction 2.

\section{Non-Bayesian Algorithms}

Study 2 replicated the three major classes of non-Bayesian algorithms identified in Study 1: joint occurrence, Fisherian, and likelihood subtraction. There was also a simpler variant of the last, the false alarm complement algorithm, which computes $1-p(D \mid-H)$ and is a shortcut for likelihood subtraction when diagnosticity (the hit rate) is high. The other new algorithms-"'total negatives," "positives times base rate," "positives times hit rate," and "hit rate minus base rate"-were only or predominantly elicited by the hybrid menu and seemed to us to be trial and error calculations. They seem to have been used in situations where the participants had no idea of how to reason from the probability or relative frequency format and tried somehow to integrate the information (such as by multiplying everything).

\section{Are Individual Inferences Menu Dependent?}

Each participant worked on each problem in three different menus. This allows us to see to what extent the cognitive algorithms and probability estimates of each individual were stable across menus. The degree of menu dependence (the sensitivity of algorithms and estimates to changes in menu) in probability formats was striking. The number of times the same algorithm could be used across the three menus is some number between 0 and 360 ( 24 problems times 15 participants). The actual number was only 16 and consisted of 10 Bayesian and 6 joint occurrence algorithms. Thus in $96 \%$ of the 360 triples, the cognitive algorithm was never the same across the three menus. In the Appendix, we illustrate this general finding through the German measles problem, which represents an "average" problem in terms of menu dependence. ${ }^{5}$ These cases reveal how helpless and inconsistent participants were when information was represented in a probability or relative frequency format.

\footnotetext{
${ }^{4}$ Study 2 was performed before Study 1 but is presented here second because it builds on the central Study 1 . In a few cases the numerical information in the problems (e.g., German measles problem) was different in the two studies.

${ }^{5}$ The German measles problem was "average" with respect to both the menu dependence of probability estimates and the menu dependence of cognitive algorithms. The average range of probability estimates in the three menus (highest minus lowest per participant) was 40.5 percentage points for all problems and 41 for the German measles problem.
} 
Table 4

Cognitive Algorithms in Study 2

\begin{tabular}{|c|c|c|c|c|c|c|c|c|}
\hline \multirow[b]{2}{*}{ Cognitive algorithm } & \multirow[b]{2}{*}{ Formal equivalent } & \multicolumn{2}{|c|}{ Information format } & \multicolumn{3}{|c|}{ Information menu } & \multirow[b]{2}{*}{ Total } & \multirow[b]{2}{*}{ \% of tota } \\
\hline & & frequency & Probability & Standard & Hybrid & Short & & \\
\hline Joint occurrence & $p(H \& D)$ & 91 & 88 & 46 & 31 & 102 & 179 & 16.6 \\
\hline Bayesian & $p(H \mid D)$ & 60 & 66 & 23 & 40 & 63 & 126 & 11.7 \\
\hline Fisherian & $p(D \mid H)$ & 46 & 45 & 41 & 50 & & 91 & 8.4 \\
\hline Adjusted Fisherian & $p(D \mid H) \pm .05$ & 20 & 29 & 20 & 29 & & 49 & 4.5 \\
\hline Multiply all & $p(D)(p(H \& D)$ & 11 & 27 & & 3 & 35 & 38 & 3.5 \\
\hline False alarm complement & $1-p(D \mid-H)$ & 17 & 20 & 37 & & & 37 & 3.4 \\
\hline Likelihood subtraction & $p(D \mid H)-p(D \mid-H)$ & 19 & 9 & 28 & & & 28 & 2.6 \\
\hline Base rate only & $p(H)$ & 14 & 10 & 14 & 10 & & 24 & 2.2 \\
\hline Total negatives & $1-p(D)$ & 10 & 7 & & 9 & 8 & 17 & 1.6 \\
\hline Positives times base rate & $p(D) p(H)$ & 7 & 7 & & 14 & & 14 & 1.3 \\
\hline Positives times hit rate & $p(D) p(D \mid H)$ & 4 & 9 & & 13 & & 13 & 1.2 \\
\hline Hit rate minus base rate & $p(D \mid H)-p(H)$ & 6 & 5 & 3 & 8 & & 11 & 1.0 \\
\hline $\begin{array}{l}\text { Less frequent agorithms } \\
\text { (<1\% of total) }\end{array}$ & & 60 & 37 & 37 & 34 & 26 & 97 & 9.0 \\
\hline Not identified & & 175 & 181 & 111 & 119 & 126 & 356 & 33.0 \\
\hline Total & & 540 & 540 & 360 & 360 & 360 & 1,080 & 100.0 \\
\hline
\end{tabular}

Note. Numbers are absolute frequencies.

Menu-dependent algorithms imply menu-dependent probability estimates. The individual cases in the Appendix are telling: Marc's estimates ranged from $0.1 \%$ to $95.7 \%$ and Oliver's from $0.5 \%$ to $100 \%$. The average range (highest minus lowest estimate) for all participants and problems was 40.5 percentage points.

\section{The Effect of Extensive Practice}

With 72 inference problems per participant, Study 2 can answer the question of whether mere practice (without feedback or instruction) increased the proportion of Bayesian algorithms. There was virtually no increase during the first three sessions, which comprised 36 tasks. Only thereafter did the proportion increase-from $.04, .07$, and .14 (standard, hybrid, and short menus, respectively) in the first three sessions to $.08, .14$, and .21 in Sessions 4 through 6 . Thus, extensive practice seems to be needed to increase the number of Bayesian responses. In Study 1, with "only" 30 problems per participant, the proportion increased slightly from .30 in the first session to .38 in the second. More generally, with respect to all cognitive algorithms, we found that when information was presented in a frequency format, our participants became more consistent in their use of cognitive algorithms with time and practice, whereas there was little if any improvement over time with probability formats. ${ }^{6}$

\section{Summary of Study 2}

Our theoretical results were that the computational complexity of Bayesian algorithms varied between the three probability menus, but not between the probability and relative frequency formats. Empirical tests showed that the actual proportion of Bayesian algorithms followed this pattern; the proportion strongly increased across menus but did not differ between the probability and the relative frequency formats, which is consistent with Predictions 2 and 4.

\section{General Discussion}

We return to our initial question: Is the mind, by design, predisposed against performing Bayesian inference? The conclusion of 25 years of heuristics-and-biases research would suggest as much. This previous research, however, has consistently neglected Feynman's (1967) insight that mathematically equivalent information formats need not be psychologically equivalent. An evolutionary point of view suggests that the mind is tuned to frequency formats, which is the information format humans encountered long before the advent of probability theory. We have combined Feynman's insight with the evolutionary argument and explored the computational implications: "Which computations are required for Bayesian inference by a given information format and menu?' Mathematically equivalent representations of information can entail computationally different Bayesian algorithms. We have argued that information representation affects cognitive algorithms in the same way. We deduced four novel predictions concerning when information

\footnotetext{
${ }^{6}$ The number of times a participant used the same cognitive algorithm (Bayesian or otherwise) in two subsequent problems with the same format and menu is a measure of temporal consistency (in a sequence of 24 problems, the number would lie between 0 and 23.) This number can be expressed as a relative frequency $c$; large values of $c$ reflect high consistency. When information was presented in frequencies (Study 1), participants became more consistent during practice, both for the standard menu (from mean consistency $c=.32$ in the first session to .49 in the second session) and for the short menu (from $c=$ .61 to .76). In contrast, when information was presented in probabilities, there was little improvement in consistency over time, regardless of the menu. For the standard probability format, $c$ was .22 for the first session and .24 for the second session of Study 1 ; in Study 2, the value was .15 in Sessions 1 to 3 and .16 in Sessions 4 to 6. The values for the short probability format were generally higher but also showed little improvement over time, shifting only from $c=.40$ to .42 (averaged across both studies).
} 
formats and menus make a difference and when they do not. Data from more than 2,800 individual problem solutions are consistent with the predictions. Frequency formats made many participants' inferences strictly conform (in terms of outcome and process) to Bayes' theorem without any teaching or instruction. These results were found for a number of inferential problems, including classic demonstrations of non-Bayesian inference such as the cab problem (Bar-Hillel, 1980; Tversky \& Kahneman, 1982) and the mammography problem (Eddy, 1982).

The results of the 15 problems in Study 1 constitute most of the data available today about Bayesian inference with frequency information. We know of only a few studies that have looked at Bayesian inference through frequency formats. Christensen-Szalanski and Beach (1982) sequentially presented symptom and disease information for 100 patients and asked participants to estimate $p$ (disease /positive). Thus, their format was mixed: natural sampling of frequencies with a single-event probability judgment (see also Gavanski \& Hui, 1992). The means from the natural sampling condition conformed better to Bayes' theorem than those from the standard probability version; however, only means-and not individual judgments or processes-were analyzed. Cosmides and Tooby (in press) constructed a dozen or so versions of the medical problem presented by Casscells et al. (1978). They converted, piece by piece, probability information into frequencies and showed how this increases, in the same pace, the proportion of Bayesian answers. They reported that when the frequency format was mixed-that is, when the information was represented in frequencies, but the single-point estimate was a single-event probability, or vice versa-the effect of the frequency format was reduced by roughly half. Their results are consistent with our theoretical framework.

At the beginning of this article, we contrasted the belief of the Enlightenment probabilists that the laws of probability theory were the laws of the mind (at least for hommes éclairés) with the belief of the proponents of the heuristics-and-biases program that the laws of probability are not the laws of the mind. We side with neither view, nor with those who have settled somewhere in between the two extremes. Both views are based on an incomplete analysis: They focus on cognitive algorithms, good or bad, without making the connection between an algorithm and the information format it has been designed for. ${ }^{7}$ Through exploration of the computational consequences of an evolutionary argument, a novel theoretical framework for understanding intuitive Bayesian inference has emerged.

We would like to emphasize that our results hold for an elementary form of Bayesian inference, with binary hypotheses and data. Medical tests involving mammograms, HIV tests, and the like are everyday examples where this elementary form of inference is of direct relevance. However, there exist other situations in which hypotheses, data, or both are multinomial or continuous and where there is not only one datum, but several. In particular, when human inference has to deal with several cues or data that are not independent, Bayesian calculations can become extremely complicated mathematically. Here, it is unlikely that frequency formats would elicit Bayesian algorithms. Rather, we suggest that in these situations "satisficing" cognitive algorithms are invoked, which can perform well in complex ecological environments. The fast and frugal algorithm described in the theory of probabilistic mental models (Gigerenzer, 1993a; Gigerenzer, Hoffrage, \& Kleinbölting, 1991) is one example; it can make inferences about unknown aspects of the real world as accurately as so-called optimal algorithms (Gigerenzer \& Goldstein, 1995).

We conclude by discussing one specific result and the general implications of the present work.

\section{Does the Use of Non-Bayesian Algorithms Follow Bayesian Intuitions?}

In applications of significance testing in the social sciences, Bayesian reasoning is sometimes used implicitly. An example would be the refusal to consider a parapsychological hypothesis in the face of an impressively small level of significance, based on a prior probability close to zero. We now ask whether our participants also used Bayesian principles in an implicit way. Does the use of non-Bayesian algorithms follow Bayesian intuitions? Joint occurrence would lead to the same result as Bayes' theorem if $p(D)=1$. (This condition never held in our tasks.) A qualitative Bayesian intuition would be "When you use joint occurrence, use it more often when $p(D)$ is large." This intuition would imply that the correlation between the magnitude of $p(D)$ and the frequency with which participants use joint occurrence should be positive. In fact, there were positive Pearson correlations of .36 in Study 1 (over 15 problems) and .47 in Study 2 (over 24 problems).

The Fisherian algorithm would lead to the same result as Bayes' theorem if $p(H) / p(D)=1$. (This condition also never held in our tasks.) A qualitative Bayesian intuition would be "When you use the Fisherian algorithm, use it more often when the probability of the data is similar to that of the event." This intuition implies that the algorithm should be used more often when $p(H) / p(D)$ is closest to one; hence, the correlation between the frequency of use and $|1-p(H) / p(D)|$ should be negative. Indeed, the Pearson correlation was -.49 in Study 1, and -.59 in Study 2.

The effect sizes of these correlations were medium to large (by J. Cohen's, 1977, definition). A similar sensitivity was reported by Ofir (1988). Thus, the use of each of these two nonBayesian algorithms appears to be grounded in a qualitative Bayesian intuition.

\footnotetext{
${ }^{7}$ That frequency formats have rarely been used is not to say that the issue of single events versus frequency has never arisen in research on Bayesian inference. For instance, Kahneman and Tversky (1982, p. 518 ) distinguished two modes of judgment, a singular mode that generates an "inside view" and a distributional one that generates an "outside view," echoing the classical distinction between the "subjective" and the "aleatory" sides of probability. Others used frequency representations to communicate information to their readers, but not to their research participants. An early example is Hammerton (1973), who chose the standard probability format to communicate information about a clinical inference task to his participants and found that their probability judgments were in disagreement with Bayes' theorem. When explaining in the article what the task's correct answer was, he switched, without comment, to a standard frequency format. Hammerton's readers, then, could easily "see" the answer, but his participants could not.
} 


\section{Alternative Accounts}

Why do judgments depend on format and menu? We started with an evolutionary argument and explored its computational consequences. What are alternative accounts?

One explanation of the striking difference between the standard probability format and the short frequency format would be that participants were presented three pieces of information in the former, but only two in the latter. The increase in Bayesian algorithms from $16 \%$ to $50 \%$ might be due simply to the smaller number of information units to be handled in the short menu. The design of Study 1, in which each format was crossed with each menu, allowed testing of this conjecture. If true, then (a) the standard frequency format should result in a proportion of Bayesian algorithms as small as that for the standard probability format and (b) differences in performance should result from the menus, and not from the formats. The empirical results, however, are inconsistent with these implications. The performances on the standard probability and standard frequency formats differed widely, and the effect of the menu largely disappeared in the frequency format.

A second alternative account would be based on a refusal to accept our notion of a "cognitive algorithm." This account would not consider our categories of Bayesian, joint occurrence, Fisherian, and so on, as cognitive algorithms but rather would construe cognitive algorithms at a higher level of abstraction. They might take the form of more general rules, such as "pick one" (i.e., always look for the one important piece of information and ignore everything else) or "always integrate everything" (i.e., always integrate all pieces of information). The interesting point is that such an account potentially eliminates menu dependence. Imagine that the cognitive algorithm is indeed "pick one." One participant might accordingly pick $p(D \mid H)$ in the standard menu, $p(H)$ in the hybrid menu, and $p(D \& H)$ in the short menu. If the pick-one rule is the cognitive algorithm applied to all of these menus, then there is no menu dependence, although it looks so from our level of analysis. Furthermore, the always-integrate rule (but not the pick-one rule) could by mere trial and error generate some proportion of Bayesian algorithms in the short menu because the number of possible combinations of two pieces of information is small. Because each participant responded to three menus, Study 2 provides the data to test the use of these two general rules. We checked whether one or both of these general rules were used independently of the menu. However, only in 12 (out of 360 ) cases were the data consistent with the pick-one rule and in 14 cases with the always-integrate rule. Thus, there was no evidence of either general rule.

Both alternative accounts could only explain the effect of menus, not formats, even if they were valid.

\section{Earlier Approaches: "Heuristics and Biases"}

The standard probability format (and its relative frequency variant) has been the most common representation of information in psychological experiments investigating whether intuitive inference follows the dictates of Bayes' theorem. The standard probability format presented our participants' inferential competencies at their worst. Specifically, the standard probability format (a) elicited the smallest number of Bayesian algo- rithms, (b) showed the least increase in Bayesian algorithms with extensive practice, (c) showed the lowest consistency in cognitive algorithms, with no improvement in consistency with practice (see Footnote 6), and (d) elicited almost all refusals to answer that we encountered (17 out of 21 in Study 1). Testing people's competencies for Bayesian inference with standard probability formats thus seems analogous to testing a pocket calculator's competence by feeding it binary numbers.

Why have so many experimental studies used the standard probability format? Part of the reason may be historical accident. There is nothing in Bayes' theorem that dictates whether the mathematical probabilities pertain to single events or to frequencies, nor is the choice of format and menus specified by the formal rules of probability. Thomas Bayes himself seemed not to have sided with either single-event probabilities or frequencies. Like his fellow Enlightenment probabilists, he blurred the distinction between warranted degrees of belief and objective frequencies by trying to combine the two (Earman, 1992). Thus, the experimental research on Bayesian inference could as well have started with frequency representations, if not for the historical accident that it became tied to Savage's (1954) agenda of bringing singular events back into the domain of probability theory. For instance, if psychological research had been inspired by behavioral ecology, foraging theory, or other ecological approaches to animal behavior in which Bayes' theorem figures prominently (e.g., Stephens \& Krebs, 1986), then the information format used in human studies might have been frequencies from the very beginning.

\section{Neglect of Base Rates}

Most earlier research has focused on prior probabilities, as has been the case with the critique of the application of Bayes' theorem since Laplace's nonchalant assumption that ignorance could be expressed as uniform priors (Daston, 1988; Earman, 1992). The major conclusion of the last two decades of research on Bayesian inference has been that people neglect base rates most of the time (e.g., Bar-Hillel, 1990; Tversky \& Kahneman, 1982). No comprehensive theory of why and when people neglect base rate information has yet been found. The present analysis can help to fill in this gap and provides novel results concerning the nature of base rate neglect.

1. Base rate information need not be attended to in frequency formats (Result 3 ). If our evolutionary argument that cognitive algorithms were designed for frequency information acquired through natural sampling is valid, then base rate neglect may come naturally when generalizing to other information representations, such as the standard probability format (Kleiter, 1994).

2. Base rate neglect is menu specific. Even within probability formats, base rate neglect is bound to specific menus. It can occur in the standard and hybrid menus, but not in the short menu. Base rate neglect is thus contingent on information menu.

3. Base rate neglect is a consequence of (at least) two different cognitive algorithms. Which cognitive algorithms entail base rate neglect? We have identified two: Fisherian (including adjusted Fisherian) and likelihood subtraction (including its shortcut version, false alarm complement). Neither of these can be elicited in the short menu. 
4. Process analysis is indispensable in establishing base rate neglect. This last point is methodological. Cognitive algorithms having in common the neglect of base rate information can nevertheless entail different probability judgments. Thus, no single probability judgment can be deduced from base rate neglect per se. The same holds for the inverse inference, from probability judgment to base rate neglect. A particular probability judgment can be produced by various algorithms, including ones that do and do not use base rates (see Birnbaum's, 1983, insightful analysis of the cab problem). Thus, base rate neglect cannot safely be inferred from a particular probability judgment, that is, from mere outcome analysis, without being double-checked by process analysis, such as "write aloud" protocols. Working through several thousand individual protocols, we observed many cases where probabilities of similar magnitude were computed by different cognitive algorithms. However, almost all previous work has relied on outcome analysis only.

\section{Representativeness Heuristic}

Tversky and Kahneman's (1974, 1982) "representativeness heuristic" has so far provided the most widely used explanation for base rate neglect in terms of a cognitive process. Judgment by representativeness means that the probability $p(H \mid D)$ is inferred from the similarity between $D$ and $H$. However, how this similarity is computed and how the inference from the similarity to a numerical probability is made have not been clarified since the notion was first proposed; "representativeness" is consequently still vague and undefined (Shanteau, 1989). Furthermore, it is not applicable to most of the problems in Studies 1 and 2, including all medical problems and the cab problem (e.g., it makes little sense to ask how representative a mother's German measles are of a child's rubella syndrome). We suggest defining the notion of representativeness by the statistical concept of the likelihood $p(D \mid H)$, a proposal made by Gigerenzer and Murray (1987, pp. 153-155). We thereby treat the representativeness heuristic as a special case of the Fisherian algorithm-namely, when the likelihood is interpreted as a degree of similarity between $D$ and $H$. If one accepts this proposal, two interesting implications follow:

1. Frequency formats suppress inference by "representativeness." We observed that use of the Fisherian algorithm decreased from 99 cases in the probability format to 55 cases in the frequency format (including the adjusted variant, see Table 3 ). This format dependency is a striking empirical result. Nothing prevented our participants from picking " $d$ out of $h$ " in the frequency format as often as picking $p(D \mid H)$ in the probability format. The decrease occurred generally in all problems, whether $p(D \mid H)$ could be interpreted as a similarity relation or not.

2. The "representativeness heuristic" is menu specific. Inferences by "representativeness" can be elicited by the standard menu, but not by the short menu (Tables 3 and 4 ). Thus, when information is acquired by natural sampling, representativeness should play no role.

If we take these two implications together, then we arrive at the result that the representativeness heuristic is most likely to be elicited when information is represented in the standard probability format. This result provides a partial answer to the unresolved question of what conditions favor particular heuristics (Brown \& Siegler, 1993).

\section{Pseudodiagnosticity}

Most of the research on Bayesian inference has focused on the neglect of base rates, but a few studies have investigated the use or neglect of $p(D \mid-H)$ in Bayesian inference. The phenomenon of people ignoring $p(D \mid-H)$ has been termed pseudodiagnosticity (Doherty \& Mynatt, 1990; Fischhoff \& BeythMarom, 1983; Ofir, 1988). The term stems from an information selection task in which participants are presented with both $p(D \mid H)$ and $p(D \mid-H)$ but tend to use only one of the two likelihoods, usually $p(D \mid H)$. Our analysis revealed that the false alarm rate was about as often neglected as the base rate. In Studies 1 and 2, the false alarm rate was neglected in $31 \%$ and 33\% of cases, the base rate in $32 \%$ and $36 \%$, respectively (these are conservative estimates because the less frequent algorithms are not counted). We were able to identify cognitive algorithms underlying this phenomenon: joint occurrence, Fisherian, and base rate only.

\section{Do Frequency Formats Influence Statistical Reasoning Beyond Bayesian Inference?}

It is interesting that most so-called biases and fallacies in probabilistic and statistical reasoning, such as base rate neglect, have been demonstrated using problems with probability formats. Can frequency formats affect other "cognitive illusions"? The evidence suggests so (see Tversky \& Kahneman, 1974). Gigerenzer et al. (1991) showed that the "overconfidence bias" (e.g., Lichtenstein et al., 1982) disappeared when participants estimated frequencies instead of single-event probabilities (see also May, 1987; Sniezek \& Buckley, 1993). This seems to be the only case aside from the present analysis of Bayesian inference where a cognitive algorithm is proposed, namely by the theory of probabilistic mental models (Gigerenzer, 1993a; Gigerenzer et al., 1991), to explain why and how format affects inferences. The theory additionally specifies conditions in which frequency judgments can be made less realistic than probability judgments.

Fiedler (1988) and Hertwig and Gigerenzer (1995) showed that the "conjunction fallacy" (Tversky \& Kahneman, 1983) in the Linda problem and similar problems largely disappeared when questions were changed from probability to frequency formats; the proportion of conjunction rule violations dropped from more than $80 \%$ to about $10 \%$ to $20 \%$ (see also Reeves \& Lockhart, 1993; Tversky \& Kahneman, 1983). As with the "overconfidence bias," this effect of frequency format is stronger and more reliable than those of all earlier so-called debiasing methods (as summarized by Fischhoff, 1982).

Koehler, Gibbs, and Hogarth ( 1994) reported that the "illusion of control" (Langer, 1975) is reduced when the single-event format is replaced by a frequency format-when participants judge a series of events rather than a single event. Teigen (1974) reported that overestimation of probabilities (e.g., What is the probability that a randomly chosen female student at the University of Bergen is above $160 \mathrm{~cm}$ tall?) changed into more realistic estimates when participants were given the opportunity to estimate frequencies (e.g., If we measure 500 female students, how many of them will be above $160 \mathrm{~cm}$ tall?). Keren and Wa- 
genaar ( 1987) observed that the "certainty effect" and the "possibility effect," two violations of utility theory, occurred less often when single gambles were replaced by repeated gambles (see aiso Keren, 1991; Montgomery \& Adelbratt, 1982). For a general discussion of these results see Ayton and Wright (1994), Gigerenzer (1991a, 1991b, 1993a, 1994), and Lopes (1981).

These converging results do have implications for how to understand so-called cognitive illusions. Were cognitive illusions due to the mind's inherent inability to reason statistically, or if they were simply the result of wishful thinking or other motivational deficits, then a frequency format should make no difference. The evidence so far, however, suggests that frequency format can make quite a difference.

\section{Some Practical Consequences}

Cognitive algorithms, Bayesian or otherwise, cannot be divorced from the information on which they operate and how that information is represented. This striking result can be made useful for teaching statistical reasoning and for human engineering in general (von Winterfeldt \& Edwards, 1986). The problems used in our studies, such as pregnancy tests, mammograms, and HIV tests, exemplify situations where Bayesian inference can help people to grasp the risks and uncertainties of a modern, technological world. However, the teaching of statistical reasoning is still a field neglected in high school mathematics education (Shaughnessy, 1992), and instruction in Bayesian inference seems to be almost nonexistent. Up until now, only a few studies have attempted to teach Bayesian inference, mainly by outcome feedback, and with little or no success (Lindeman, van den Brink, \& Hoogstraten, 1988; Peterson, DuCharme, \& Edwards, 1968; Schaefer, 1976).

The present framework suggests an effective way to teach Bayesian inference and statistical reasoning generally. The lesson of our studies is to teach representations instead of rules, that is, to teach people how to translate probabilities into frequency representations rather than how to insert probabilities into Equation 1. In light of our results concerning how Bayesian inference can be improved without any instruction, tutoring systems that enhance the idea of frequency representations with instruction, explanation, and visual aids hold out the promise of still greater success. Frequency representations can help people "see" the answer, appealing to a "sort of instinct" at which Laplace and the Enlightenment probabilists hinted.

\section{References}

Ajzen, I. (1977). Intuitive theories of events and the effects of baserate information on predictions. Journal of Personality and Social Psychology, 35, 303-314.

Allais, M. (1953). Le comportement de l'homme rationnel devant le risque: Critique des postulats et axiomes de l'Ecole Américaine [The behavior of rational man with respect to risk: A criticism of the postulates and axioms of the American school]. Econometrica, 21, 503546.

Ayton, P., \& Wright, G. ( 1994). Subjective probability: What should we believe? In G. Wright \& P. Ayton (Eds.), Subjective probability (pp. 163-184). New York: Wiley.

Bar-Hillel, M. (1980). The base-rate fallacy in probability judgments. Acta Psychologica, 44, 211-233.

Bar-Hillel, M. (1990). Back to base rates. In R. M. Hogarth (Ed.), In- sights in decision making (pp. 309-330). Chicago: University of Chicago Press.

Barsalou, L. W., \& Ross, B. H. (1986). The role of automatic and strategic processing in sensitivity to superordinate and property frequency. Journal of Experimental Psychology: Learning, Memory, and Cognition, 12, 116-134.

Bayes, T. (1763). An essay towards solving a problem in the doctrine of chances. Philosophical Transactions of the Royal Society of London, $53,370-418$.

Birnbaum, M. H. (1983). Base rates in Bayesian inference: Signal detection analysis of the cab problem. American Journal of Psychology, 96, 85-94.

Boole, G. (1958). An investigation of the laws of thought. New York: Dover. (Original work published 1854 )

Borgida, E., \& Brekke, N. (1981). The base-rate fallacy in attribution and prediction. In J. H. Harvey, W. J. Ickes, \& R. F. Kidd (Eds.), New directions in attribution research (pp. 66-95). Hillsdale, NJ: Erlbaum.

Brown, N. R., \& Siegler, R. S. (1993). Metrics and mappings: A framework for understanding real-world quantitative estimation. Psychological Review, 100, 511-534.

Brunswik, E. (1939). Probability as a determiner of rat behavior. Journal of Experimental Psychology, 25, 175-197.

Brunswik, E. (1955). Representative design and probabilistic theory in a functional psychology. Psychological Review, 62, 193-217.

Casscells, W., Schoenberger, A., \& Grayboys, T. (1978). Interpretation by physicians of clinical laboratory results. New England Journal of Medicine, 299, 999-1000.

Christensen-Szalanski, J. J. J., \& Beach, L. R. (1982). Experience and the base-rate fallacy. Organizational Behavior and Human Performance, 29, 270-278.

Cohen, J. (1977). Statistical power analysis for the behavioral sciences (2nd ed.). New York: Academic Press.

Cohen, L. J. (1986). The dialogue of reason. Oxford, England: Clarendon Press.

Cosmides, L., \& Tooby, J. (in press). Are humans good intuitive statisticians after all? Rethinking some conclusions from the literature on judgment under uncertainty. Cognition.

Daston, L. J. (1981). Mathematics and the moral sciences: The rise and fall of the probability of judgments, 1785-1840. In H. N. Jahnke \& M. Otte (Eds.), Epistemological and social problems of the sciences in the early nineteenth century (pp. 287-309). Dordrecht, The Netherlands: D. Reidel.

Daston, L. J. (1988). Classical probability in the Enlightenment. Princeton, NJ: Princeton University Press.

Doherty, M. E., \& Mynatt, C. R. (1990). Inattention to $\mathrm{p}(\mathrm{H})$ and to $\mathrm{p}(\mathrm{D} \mid-\mathrm{H})$ : A converging operation. Acta Psychologica, 75, 1-11.

Earman, J. (1992). Bayes or bust? A critical examination of Bayesian confirmation theory. Cambridge, MA: MIT Press.

Eddy, D. M. (1982). Probabilistic reasoning in clinical medicine: Problems and opportunities. In D. Kahneman, P. Slovic, \& A. Tversky (Eds.), Judgment under uncertainty: Heuristics and biases (pp. 249267). Cambridge, England: Cambridge University Press.

Edwards, W. (1968). Conservatism in human information processing. In B. Kleinmuntz (Ed.), Formal representation of human judgment (pp. 17-52). New York: Wiley.

Edwards, W., \& von Winterfeldt, D. (1986). On cognitive illusions and their implications. In H. R. Arkes \& K. R. Hammond (Eds.), Judgment and decision making. Cambridge, England: Cambridge University Press.

Ericsson, K. A., \& Simon, H. A. (1984). Protocol analysis: Verbal reports as data. Cambridge, MA: MIT Press.

Feynman, R. (1967). The character of physical law. Cambridge, MA: MIT Press.

Fiedler, K. ( 1988). The dependence of the conjunction fallacy on subtle linguistic factors. Psychological Research, 50, 123-129. 
Fischhoff, B. ( 1982). Debiasing. In D. Kahneman, P. Slovic, \& A. Tversky (Eds.), Judgment under uncertainty: Heuristics and biases (pp. 422-444). Cambridge, England: Cambridge University Press.

Fischhoff, B., \& Beyth-Marom, R. (1983). Hypothesis evaluation from a Bayesian perspective. Psychological Review, 90, 239-260.

Fisher, R. A. (1955). Statistical methods and scientific induction. Journal of the Royal Statistical Society, Series B, 17, 69-78.

Gallistel, C. R. (1990). The organization of learning. Cambridge, MA: MIT Press.

Gavanski, I., \& Hui, C. (1992). Natural sample spaces and uncertain belief. Journal of Personality and Social Psychology, 63, 766-780.

Gigerenzer, G. (1991a). From tools to theories: A heuristic of discovery in cognitive psychology. Psychological Review, 98, 254-267.

Gigerenzer, G. (1991b). How to make cognitive illusions disappear: Beyond "heuristics and biases." In W. Stroebe \& M. Hewstone (Eds.), European Review of Social Psychology, 2, 83-115.

Gigerenzer, G. (1993a). The bounded rationality of probabilistic mental models. In K. I. Manktelow \& D. E. Over (Eds.), Rationality: Psychological and philosophical perspectives (pp. 284-313). London: Routledge.

Gigerenzer, G. (1993b). The superego, the ego, and the id in statistical reasoning. In G. Keren \& C. Lewis (Eds.), $A$ handbook for data analysis in the behavioral sciences: Methodological issues (pp. 313-339). Hillsdale, NJ: Erlbaum.

Gigerenzer, G. (1994). Why the distinction between single-event probabilities and frequencies is relevant for psychology (and vice versa). In G. Wright \& P. Ayton (Eds.), Subjective probability (pp. 129162). New York: Wiley.

Gigerenzer, G., \& Goldstein, D. G. (1995). Reasoning the fast and frugal way: Models of bounded rationality. Manuscript submitted for publication.

Gigerenzer, G., Hell, W., \& Blank, H. ( 1988). Presentation and content: The use of base rates as a continuous variable. Journal of Experimental Psychology: Human Perception and Performance, 14, 513-525.

Gigerenzer, G., Hoffrage, U., \& Kleinbölting, H. (1991). Probabilistic mental models: A Brunswikian theory of confidence. Psychological Review, 98, 506-528.

Gigerenzer, G., \& Murray, D. J. (1987). Cognition as intuitive statistics. Hillsdale, NJ: Erlbaum.

Gigerenzer, G., Swijtink, Z., Porter, T., Daston, L., Beatty, J., \& Krüger, L. (1989). The empire of chance: How probability changed science and everyday life. Cambridge, England: Cambridge University Press.

Gould, S. J. (1992). Bully for brontosaurus: Further reflections in natural history. New York: Penguin Books.

Hammerton, M. (1973). A case of radical probability estimation. Journal of Experimental Psychology, 101, 252-254.

Hammond, K. R. (1990). Functionalism and illusionism: Can integration be usefully achieved? In R. M. Hogarth (Ed.), Insights in decision making (pp. 227-261). Chicago: University of Chicago Press.

Hasher, L., \& Zacks, R. T. (1979). Automatic and effortful processes in memory. Journal of Experimental Psychology: General, 108, 356388.

Hertwig, R., \& Gigerenzer, G. (1995). The chain of reasoning in the conjunction task. Unpublished manuscript.

Hintzman, D. L. (1976). Repetition and memory. In G. H. Bower (Ed.), The psychology of learning and motivation (Vol. 10, pp. 4791). New York: Academic Press.

Hume, D. (1951). A treatise of human nature (L. A. Selby-Bigge, Ed.). Oxford, England: Clarendon Press. (Original work published 1739)

Kahneman, D., \& Tversky, A. (1972). Subjective probability: A judgment of representativeness. Cognitive Psychology, 3, 430-454.

Kahneman, D., \& Tversky, A. (1973). On the psychology of prediction. Psychological Review, 80, 237-251.

Kahneman, D., \& Tversky, A. (1982). Variants of uncertainty. In D. Kahneman, P. Slovic, \& A. Tversky (Eds.), Judgment under un- certainty: Heuristics and biases (pp. 509-520). Cambridge, England: Cambridge University Press.

Keren, G. (1991). Additional tests of utility theory under unique and repeated conditions. Journal of Behavioral Decision Making, 4, 297304.

Keren, G., \& Wagenaar, W. A. (1987). Violation of utility theory in unique and repeated gambles. Journal of Experimental Psychology: Learning, Memory, and Cognition, 13, 387-391.

Kleiter, G. D. (1994). Natural sampling: Rationality without base rates. In G. H. Fischer \& D. Laming (Eds.), Contributions to mathematical psychology, psychometrics, and methodology (pp. 375-388). New York: Springer.

Koehler, J. J. (in press) . The base rate fallacy reconsidered: Descriptive, normative and methodological challenges. Behavioral and Brain Sciences.

Koehler, J. J., Gibbs, B. J., \& Hogarth, R. M. (1994). Shattering the illusion of control: Multi-shot versus single-shot gambles. Journal of Behavioral Decision Making, 7, 183-191.

Kosslyn, S. M., \& Pomerantz, J. R. (1977). Imagery, propositions, and the form of internal representations. Cognitive Psychology, 9, 52-76.

Kroznick, J. A., Li, F., \& Lehman, D. R. (1990). Conversational conventions, order of information acquisition, and the effect of base rates and individuating information on judgments. Journal of Personality and Social Psychology, 59, 1140-1152.

Langer, E. J. (1975). The illusion of control. Journal of Personality and Social Psychology, 32, 311-328.

Laplace, P.-S. (1951). A philosophical essay on probabilities (F. W. Truscott \& F. L. Emory, Trans.). New York: Dover. (Original work published 1814)

Larkin, J., \& Simon, H. (1987). Why a diagram is (sometimes) worth ten thousand words. Cognitive Science, 11, 65-99.

Lichtenstein, S., Fischhoff, B., \& Phillips, L. D. (1982). Calibration of probabilities: The state of the art to 1980 . In D. Kahneman, P. Slovic, \& A. Tversky (Eds.), Judgment under uncertainty: Heuristics and biases (pp. 306-334). Cambridge, England: Cambridge University Press.

Lindeman, S. T., van den Brink, W. P., \& Hoogstraten, J. ( 1988). Effect of feedback on base-rate utilization. Perceptual and Motor Skills, 67. 343-350.

Lopes, L. L. (1981). Decision making in the short run. Journal of Experimental Psychology: Human Learning and Memory, 7, 377-385.

Lopes, L. L. (1991). The rhetoric of irrationality. Theory and Psychology, 1, 65-82.

Lyon, D., \& Slovic, P. ( 1976). Dominance of accuracy information and neglect of base rates in probability estimation. Acta Psychologica, 40, 287-298.

Marr, D. (1982). Vision: A computational investigation into the human representation and processing of visual information. San Francisco: Freeman

May, R. S. (1987). Realismus von subjektiven Wahrscheinlichkeiten. Eine kognitionspsychologische analyse inferentieller prozesse beim over-confidence-phänomen [Calibration of subjective probabilities: A cognitive analysis of inference processes in overconfidence ]. Frankfurt, Germany: Lang.

McKenzie, C. R. (1994). The accuracy of intuitive judgment strategies: Covariation assessment and Bayesian inference. Cognitive Psychology, 26, 209-239.

Medin, D. L., Wattenmaker, W. D., \& Michalski, R. S. (1987). Constraints and preferences in inductive learning: An experimental study of human and machine performance. Cognitive Science, 11, 299339.

Montgomery, H., \& Adelbratt, T. (1982). Gambling decisions and information about expected value. Organizational Behavior and $\mathrm{Hu}$ man Performance, 29, 39-57.

Nisbett, R. E., \& Wilson, T. D. (1977). Telling more than we can know: 
Verbal reports on mental processes. Psychological Review, 84, 231259.

Oakes, M. (1986). Statistical inference: A commentary for the social and behavioral sciences. New York: Wiley.

Ofir, C. (1988). Pseudodiagnosticity in judgment under uncertainty. Organizational Behavior and Human Decision Processes, 42, 343363.

Peterson, C. R., DuCharme, W. M., \& Edwards, W. (1968). Sampling distributions and probability revision. Journal of Experimental Psychology, 76, 236-243.

Phillips, L. D., \& Edwards, W. (1966). Conservatism in a simple probability model inference task. Journal of Experimental Psychology, 72, 346-354.

Piaget, J., \& Inhelder, B. (1975). The origin of the idea of chance in children. New York: Norton. (Original work published 1951)

Pylyshyn, Z. W. (1973). What the mind's eye tells the mind's brain: A critique of mental imagery. Psychological Bulletin, 80, 1-24.

Real, L. A. (1991, August). Animal choice behavior and the evolution of cognitive architecture. Science, 253, 980-986.

Real, L. A., \& Caraco, T. (1986). Risk and foraging in stochastic environments: Theory and evidence. Annual Review of Ecology and Systematics, 17, 371-390.

Reeves, T., \& Lockhart, R. (1993). Distributional versus singular approaches to probability and errors in probabilistic reasoning. Journal of Experimental Psychology: General, 122, 207-226.

Rouanet, H. (1961). Études de décisions expérimentales et calcul de probabilités. [Studies of experimental decision making and the probability calculus] In Colloques internationaux du centre national de la recherche scientifique (pp. 33-43). Paris: Éditions du Centre National de la Recherche Scientifique.

Sahlin, N. (1993). On higher order beliefs. In J. Dubucs (Ed.), Philosophy of probability (pp. 13-34). Dordrecht, The Netherlands: Kluwer Academic.

Savage, L. J. (1954). The foundations of statistics. New York: Wiley.

Schaefer, R. E. (1976). The evaluation of individual and aggregated subjective probability distributions. Organizational Behavior and Human Performance, 17, 199-210.

Scholz, R. W. ( 1987). Cognitive strategies in stochastic thinking. Dordrecht, The Netherlands: D. Reidel.
Schum, D. A. (1994). The evidential foundations of probabilistic reasoning. New York: Wiley.

Sedlmeier, P., Hertwig, R., \& Gigerenzer, G. (1995). Is " $R$ " more likely in the first or second position? Availability, letter class, and neural network models. Manuscript submitted for publication.

Shanks, D. ( 1991 ). A connectionist account of base-rate biases in categorization. Connection Science, 3(2), 143-162.

Shanteau, J. (1989). Cognitive heuristics and biases in behavioral auditing: Review, comments and observations. Accounting Organizations and Society, 14(1/2), 165-177.

Shaughnessy, J. M. (1992). Research on probability and statistics: Reflections and directions. In D. A. Grouws (Ed.), Handbook of research on mathematical teaching and learning (pp. 465-499). New York: Macmillan.

Simon, H. A. (1956), Rational choice and the structure of the environment. Psychological Review, 63, 129-138.

Sniezek, J. A., \& Buckley, T. (1993). Decision errors made by individuals and groups. In N. J. Castellan (Ed.), Individual and group decision making. Hillsdale, NJ: Erlbaum.

Stephens, D. W., \& Krebs, J. R. (1986), Foraging theory. Princeton, NJ: Princeton University Press.

Stigler, J. W. (1984). The effect of abacus training on Chinese children's mental calculation. Cognitive Psychology, 16, 145-176.

Teigen, K. H. (1974), Overestimation of subjective probabilities. Scandinavian Journal of Psychology, 15, 56-62.

Tversky, A., \& Kahneman, D. (1974, September). Judgment under uncertainty: Heuristics and biases. Science, 185, 1124-1131.

Tversky, A., \& Kahneman, D. (1982). Evidential impact of base rates. In D. Kahneman, P. Slovic, \& A. Tversky (Eds.), Judgment under uncertainty: Heuristics and biases (pp. 153-160). Cambridge, England: Cambridge University Press.

Tversky, A., \& Kahneman, D. (1983). Extensional versus intuitive reasoning: The conjunction fallacy in probability judgment. Psychological Review, 90, 293-315.

von Winterfeldt, D., \& Edwards, W. (1986). Decision analysis and behavioral research. Cambridge, England: Cambridge University Press.

Wallsten, T. S. (1983). The theoretical status of judgmental heuristics. In R. W. Scholz (Ed.), Decision making under uncertainty (pp. 2139). Amsterdam: Elsevier (North-Holland). 


\section{Appendix \\ Instability of Judgments and Cognitive Algorithms Across Menus in Probability Formats}

In Study 2, participants received each problem three times, once in each of the three menus (standard, hybrid, and short; see text). The following 4 participants (from a total of 15 ) illustrate how both the numerical probability estimates and the cognitive algorithms varied across the three menus. The German measles problem, in which the task was to estimate the probability $p(H \mid D)$ of severe prenatal damage in the child $(H)$ if the mother had German measles during pregnancy $(D)$, is used as an example. In the standard menu, the information (probabilities expressed in percentages) was $p(H)=0.5 \%, p(D \mid H)=$ $40 \%$, and $p(D \mid-H)=0.01 \%$; in the hybrid menu, $p(H)=0.5 \%$, $p(D \mid H)=40 \%$, and $p(D)=0.21 \%$; and in the short menu, $p(D)=$ $0.21 \%$ and $P(D \& H)=0.2 \%$. Note that the information in all three menus is equivalent in the sense that it would lead to the same probability $p(H \mid D)$.

\section{Rüdiger B. (Age 22), Management}

In the standard menu, Rüdiger focused on $p(D \mid H)$, explaining that because a child of an infected mother is at such high risk $(40 \%)$, his estimate would accordingly be high. He adjusted $p(D \mid H)$ by $5 \%$, and estimated the posterior probability of severe prenatal damage as $35 \%$ (adjusted Fisherian). In the hybrid menu, he picked the base rate and estimated the same probability as $0.5 \%$, with the argument that $p(D \mid H)$ and $p(D)$ "are without significance" (base rate only). In the short menu, he picked $p(H \& D)$ and estimated $0.2 \%$ because "this is the information that specifies the probability of severe damage in the child. The percentage of infected mothers, however, is irrelevant" (joint occurrence).

\section{Marc P. (Age 22), Biology}

In the standard menu, Marc used only $p(D \mid H)$ and estimated $40 \%$ (Fisherian). In retrospect, he noticed that he forgot to include $p(D \mid-H)$, but he did not mention $p(H)$. In the hybrid menu, his answer was $0.1 \%$, which he calculated by multiplying $p(D)$ with $p(D \mid H)$ and rounding the result (positives times hit rate). In the short menu, he reasoned that $0.21 \%$ of all pregnant women do have German measles, but because German measles and severe damage co-occur only in $0.2 \%$ cases, $0.01 \%$ children would remain without damage. He set himself the intermediate goal of determining the probability of there being no prenatal damage given German measles. He first translated the probabilities into frequencies, obtaining the result of 10 out of 210 children. He then converted this frequency back into a single-event probability, which he calculated as $4.3 \%$ (a minor calculation error). He concluded, therefore, that the posterior probability of severe prenatal damage was 95.7\% (Bayesian). In the short menu, his reasoning turned Bayesian.

\section{Silke H. (Age 20), Biology}

In the standard menu, Silke started with the base rate, then calculated the proportion $p(D \mid H)$ of the base rate with an answer of $0.2 \%$ (joint occurrence). In the hybrid menu, she used all of the information. She first multiplied $p(H)$ by $p(D)$, that is, the probability of prenatal damage by the probability of German measles during pregnancy, and then determined the proportion $p(D \mid H)$ of this figure, which resulted in the answer $0.04 \%$ (multiply all). In the short menu, she used the same algorithm again and computed the proportion $p(H \& D)$ of $p(D)$, which again lead to the answer $0.04 \%$.

\section{Oliver G. (Age 22), German Literature}

In the standard menu, Oliver stated that the "correlation between not having damage and nevertheless having measles," as he paraphrased $p(D \mid-H)$, was the only relevant information. He calculated 1 $p(D \mid-H)=99.99 \%$ and rounded to $100 \%$, which was his estimate (false alarm complement). In the hybrid menu, he concluded that the only relevant information was the base rate of severe prenatal damage, and his estimate consequently dropped to $0.5 \%$ (base rate only). In the short menu, he determined the proportion of severe damage and measles in all cases with German measies, which led him to the Bayesian answer of $95.3 \%$.

Received April 27, 1994

Revision received November 29, 1994 Accepted February 27, 1995 